\title{
A theory of spontaneous tropical cyclogenesis from
}

\author{
quasi-random convection \\ Hao Fu* and Morgan O'Neill \\ Department of Earth System Science, Stanford University
}

* email: haofu@stanford.edu

This is a preprint for EarthArXiv. The manuscript has been submitted to Journal of the Atmospheric Sciences. Subsequent versions may have slight modifications. The authors welcome feedbacks from anyone who reads the manuscript. 


\title{
A theory of spontaneous tropical cyclogenesis from quasi-random
}

\author{
Hao $\mathrm{Fu}^{*}$ and Morgan O'Neill \\ Department of Earth System Science, Stanford University, California
}

${ }_{5}^{*}$ Corresponding author address: Hao Fu, 473 Via Ortega, Stanford, CA 94305.

•E-mail: haofu@stanford.edu 


\section{ABSTRACT}

How the cumulus clouds organize into a tropical cyclone remains poorly understood. The difficulty lies in that the deep convection is noisy at the kilometer scale, but follows the physical feedbacks at the mesoscale. We build a barotropic numerical model to understand the interaction of the stochastic and deterministic processes in the genesis of a tropical depression. Deep convection is represented as a multitude of isolated convergence forcing. The convection is assigned to distribute randomly at the small scale. At the mesoscale, convection is preferentially seeded to regions with a high spatially-filtered vertical vorticity. The preferential seeding mimics the physical feedbacks, and the filter implicitly represents the nonlocal convective triggering by gravity wave and cold pool. The result shows that the early-stage evolution is dominated by random vortex tube stretching. Subsequently, the regions where repetitive stretching occurs become vortex clusters, and induce more convection around them. The collision and coalescence between vortex clusters lead to a major vortex, which accelerates the growth by the preferential seeding. This physical picture agrees with a cloud-permitting simulation of spontaneous tropical cyclogenesis over uniform sea surface temperature. A theoretical model with approximate analytical solution is presented to depict the full evolution process. 


\section{Introduction}

Convection is stochastic at the small scale $(\lesssim 10 \mathrm{~km})$ and in quasi-equilibrium with the environment at the large scale $\left(\gtrsim 10^{3} \mathrm{~km}\right)$ where a sufficient amount of clouds is embedded. However, tropical cyclogenesis requires clouds to spontaneously organize via the $10 \sim 10^{3} \mathrm{~km}$ physical feedback of convection with Ekman pumping (Charney and Eliassen 1964), wind-induced surface flux (Emanuel 1986; Raymond et al. 2007), moisture (Montgomery et al. 2006; Dunkerton et al. 2009; Bell and Montgomery 2019), and radiation (Davis 2015; Muller and Romps 2018; Yang and Tan 2020; Ruppert et al. 2020). The interaction between the small-scale and mesoscale processes (Fang and Zhang 2011), the interaction between the stochastic and deterministic factors (Craig and Mack 2013), as well as the eddying motion (Ritchie and Holland 1997; Schecter and Dunkerton 2009; Davis 2015), render the basic challenges in understanding tropical cyclogenesis. To the authors' knowledge, no theoretical framework can address all the three factors, which we believe are intrinsically linked to each other.

The stochastic component can lead to a nonuniform water vapor and vorticity field via the accumulation of random events (Hottovy and Stechmann 2015; Fu and O'Neill 2021). These anomalies do not quickly disappear after individual convective events, and lucky places can receive multiple updrafts to become a moist and high-vorticity region. However, once the stochastic process leads to a strong enough disturbance, the local physical feedbacks kick in.

Wing et al. (2016) showed that the longwave cloud-radiation feedback and the wind-induced surface heat flux feedback (WISHE) are the main positive diabatic feedbacks that favor tropical cyclogenesis, and the advective transport of environmental air to the convective region is a negative feedback. In a moister region, the larger high cloud cover due to more vigorous convection reduces radiative cooling, and drives a secondary circulation. On one hand, the ascending branch of the 
circulation moistens the troposphere and favors deep convection (Ruppert et al. 2020). On the other hand, the inflow branch of the circulation transports the surrounding air inside. Dynamically, the inflow induces the convergence of angular momentum, which directly spins up the middle layer (Ruppert et al. 2020). Thermodynamically, if the inflow is primarily in the middle troposphere (top-heavy updraft profile), the drier air is entrained, which disfavors deep convection. Such a positive gross moist stability scenario (Neelin and Held 1987) was reported by Wing et al. (2016). If the inflow is primarily in the boundary layer (bottom-heavy), high enthalpy air is entrained and favors deep convection, as was reported by Ruppert et al. (2020). The deep convection heats the middle layer due to condensation, and cools the lower layer due to the evaporative cooling of precipitation, rendering a middle level vortex at the early stage (Houze Jr et al. 2009). The growth of middle level vortex is accompanied by higher surface wind, which enhances surface heat flux and favors deep convection (Wing et al. 2016). In addition, the lower level circulation causes Ekman pumping, which has dual roles. It favors deep convection and therefore indirectly spins up the lower troposphere in a high conditional instability region, but it spins down the lower troposphere in a low conditional instability region (Ooyama 1969; Schecter and Dunkerton 2009; Schecter 2011).

Though these physical feedbacks work locally, the nonlocal triggering by gravity wave and cold pool could diffuse the local feedback signal (Mapes 1993; Windmiller and Craig 2019; Yang 2020). As a result, we hypothesize that the feedbacks effectively work at the mesoscale. The two nonlocal triggering processes are reviewed below.

Mapes (1993) considered that the second baroclinic mode gravity wave, which is excited by the middle level evaporative cooling of precipitating convection (and especially mesoscale convective system), can trigger new convection by reducing the low-level stratification. It may render a $\sim 200$ $\mathrm{km}$ length scale for convective organization. When including the planetary rotation, the cooling 
anomaly is restored within the deformation radius and can accumulate (Liu and Moncrieff 2004). Brenowitz et al. (2016) applied the wave-trigger thinking to modelling a Kelvin wave with a onedimensional (1D) shallow water model. They let the convective mass flux depend on a spatially filtered low-level convergence. Linear stability analysis showed that a most unstable wavelength exists and it increases with the filter length. Liu et al. (2019) showed that a temporal filter on the convective mass flux yields a similar effect for modelling a Kelvin wave.

The boundary layer cold pool (gravity current) is the evaporation-driven thunderstorm outflow, which yields a shorter nonlocal triggering length scale. The cold pools can non-locally trigger convection by dynamical lifting, and by accumulating water vapor at their gust fronts (Tompkins 2001; Jeevanjee and Romps 2013; Langhans and Romps 2015; Torri and Kuang 2019). As the convective cluster grows, the incoherent cold pools could fuse into a $\sim 100 \mathrm{~km}$ radius mesoscale cold pool. The convective-and-non-convective interface appears as a quasi-circular mesoscale front, balanced by the boundary layer inflow (Windmiller and Hohenegger 2019). This shock formation phenomenon is a deviation from pure "diffusion".

The physical feedbacks and the diffusive phenomenon of convective activity inspire people to model the non-rotating convective organization as a coarsening process, which is the evolution of a reaction-diffusion equation that could lead to pattern formation (Cross and Hohenberg 1993; Craig and Mack 2013; Windmiller and Craig 2019; Ahmed and Neelin 2019). Though the coarsening model might be extended to study tropical cyclogenesis and considered as a model of the mesoscale field, the interaction of the mesoscale with the stochastic cloud-scale is absent within the simple reaction-diffusion framework. In addition, the eddying behavior of the convective patches can lead to mutual shearing and merger (Ritchie and Holland 1997; Schecter and Dunkerton 2009; Davis 2015). Its contribution to convective organization also lacks a theoretical model. 
In this paper, we present a novel one-layer barotropic model to study the organization of rotating moist convection. To represent the mixed stochastic-deterministic process, isolated short-lived convection is randomly seeded based on a filtered vorticity field. The filter kernel is Gaussian, with a fixed length scale that implicitly represents the nonlocal convective trigger by gravity wave and cold pool. To the authors' knowledge, this is the first simple model that can depict both the random superposition of cloud-generated vorticity and the mesoscale physical feedback. This is a prototype model of tropical cyclogenesis in the more realistic rotating radiative convective equilibrium (RRCE) setup (Bretherton et al. 2005; Khairoutdinov and Emanuel 2013; Wing et al. 2016; Muller and Romps 2018; Carstens and Wing 2020; Yang and Tan 2020). The RRCE is an idealized cloud-permitting simulation configuration that sets uniform sea surface temperature in a doubly periodic domain, and without mean flow. It is a suitable tool to study mesoscale processes, because the large-scale control is eliminated. We focus on the formation of tropical depression (maximum surface wind speed smaller than $17 \mathrm{~m} \mathrm{~s}^{-1}$, AMS-Glossary 2012), which is the first stage in tropical cyclogenesis (Montgomery et al. 2006). The key question is how a tropical depression grows out of the noisy convection.

This paper is organized in the following way. Section 2 introduces the one-layer (barotropic) model and its simulation result. Section 3 introduces the theoretical model. The theory is benchmarked with the sensitivity tests of the barotropic simulation in section 4 , and a single case of cloud-permitting simulation in section 5. Section 6 concludes the paper. 


\section{The barotropic model}

\section{a. The governing equations}

The dynamical core is a weak temperature gradient model, which is essentially a divergent barotropic model forced by episodic convection (Sobel et al. 2001). We let it depict the low-mid troposphere $(\sim 1-6) \mathrm{km}$. The vorticity dynamics is almost identical to a shallow water model, given the Froude number (flow velocity over gravity wave speed) is small, and the domain width $L$ is much smaller than the Rossby deformation radius $L_{R}$. Both criteria have been shown to be satisfied in the tropical depression stage (Enagonio and Montgomery 2001; Fu and O'Neill 2021). In this system, the domain-integrated vertical absolute vorticity $\omega_{a}$ is conserved, so the TC genesis is essentially an aggregation of a finite amount of absolute vorticity.

The "mesoscale" used in this paper denotes a "statistical mesoscale". It is defined as the length scale at which the physical feedbacks have a deterministic signal, though substantial fluctuation can occur due to the limited number of clouds. For tropical cyclogenesis, we judge that the "statistical mesoscale" roughly overlaps with the "dynamical mesoscale" where the stratification is strong and the background rotation is moderate (not too strong to suppress the momentum advection) (Riley and Lelong 2000). This is illustrated in Fig. 1. The strong stratification makes the gravity wave adjustment fast enough and guarantees the weak temperature gradient approximation.

The vorticity equation is:

$$
\frac{\partial \omega}{\partial t}+\mathbf{u} \cdot \nabla_{h} \omega=-\left(\omega+f_{0}\right) \delta,
$$

with

$$
\delta=\delta_{u}+\delta_{\text {rad }}+\delta_{E},
$$

$$
\mathbf{u}=\mathbf{u}_{\delta}+\mathbf{u}_{\omega},
$$




$$
\delta=\nabla \cdot \mathbf{u}_{\delta} \quad \text { and } \quad \omega=\mathbf{k} \cdot\left(\nabla \times \mathbf{u}_{\omega}\right)
$$

Here $\nabla_{h}=\mathbf{i} \partial / \partial x+\mathbf{j} \partial / \partial y$ is the horizontal gradient operator, $\omega$ is vertical relative vorticity, $\mathbf{u}$ is horizontal velocity, $\mathbf{u}_{\delta}$ is the divergent flow, $\mathbf{u}_{\omega}$ is the rotational flow, $\delta$ is the divergence, $\delta_{u}$ is the divergence due to deep convective updraft (negative value), $\delta_{r a d}$ is the divergence due to radiative cooling (positive value) and $\delta_{E}$ is the divergence due to Ekman pumping or suction. Here we follow Sansón and Van Heijst (2000) to consider the stretching/squashing of the total absolute vorticity in Ekman spin down, rather than the classic linear formula that only considers the planetary vorticity part (Vallis 2017).

The deep convection entrains lower and mid tropospheric mass and adds mass to the upper level. We represent it with a quasi-random seeding of convergence pulses, which is an update from the previous works that use random seeding in the whole domain (Vallis et al. 1997; Showman 2007; O'Neill et al. 2016), or in a certain region (Fu and O'Neill 2021). The randomness itself is a parameterization of the convective trigger by the noisy cold pool and gravity wave induced by other clouds, which are explicitly considered in some 2D one-layer models instead (Yang and Ingersoll 2013; Haerter et al. 2019; Haerter 2019; Yang 2020). For every $\Delta t$ time, a new updraft is seeded to the domain. The quasi-random seeding means, we let the probability for each position to get an updraft be measured by updraft number density $\rho_{u}(\mathbf{x}, t)$, which will be discussed shortly afterwards. The expression of $\delta_{u}$ is contributed by multiple updrafts:

$$
\delta_{u}=\sum_{n=1}^{\infty} \delta_{u m} \exp \left[-\frac{\left(\mathbf{x}-\mathbf{x}_{n}\right)^{2}}{r_{u}^{2}}-\frac{\left(t-t_{n}\right)^{2}}{\tau_{u}^{2}}\right],
$$

where $\delta_{u m}$ (negative constant) is the peak divergence of an updraft, $\mathbf{x}_{n}$ is the position vector of the $n^{\text {th }}$ updraft which follows the wind, $t_{n}$ is the peak time of the $n^{\text {th }}$ updraft, $r_{u}$ is the updraft radius scale, and $\tau_{u}$ is the updraft duration time scale. The averaged accumulated convergence 
within the radius $r_{u}$ is defined as $\Delta h / H=\delta_{u m} \tau_{u}$, which takes a negative value. The $\Delta h$ (negative value) is the air column thickness loss in a convective event, and $H \sim 5 \mathrm{~km}$ is the basic state layer thickness. The $\delta_{\text {rad }}$ is set to be uniform, which instantaneously balances all the updraft divergence synchronously:

$$
\delta_{\text {rad }} \approx-\delta_{0}=-L^{-2} \iint \delta_{u} d \mathbf{x} .
$$

Here $\delta_{0}$ is the domain averaged updraft-induced divergence (negative), around which $-\delta_{\text {rad }}$ fluctuates. The $\delta_{\text {rad }}$ can be linked to radiative cooling rate $Q_{\text {rad }}$ (unit: $\mathrm{K} \mathrm{s}^{-1}$ ):

$$
\delta_{r a d}=-\frac{Q_{r a d}}{\Delta \theta},
$$

where $\Delta \theta$ is the potential temperature difference across the $1-6 \mathrm{~km}$ height layer. The $\delta_{E}$ is calculated with the simple laminar Ekman layer model:

$$
\delta_{E}=\mathrm{E} \omega \quad \text { with } \quad \mathrm{E}=\frac{h_{E}}{2 H} .
$$

Here $\mathrm{E}$ is Ekman number which represents the vorticity-divergence (spin down) relation, and $h_{E}$ is the Ekman layer depth. Equation (8) is less accurate than the Ekman layer model that uses bulk aerodynamic formula (e.g. Schecter and Dunkerton 2009), but is more analytically tractable.

We design a quasi-stochastic convective scheme, where the local cloud population depends on the mesoscale vorticity. The anomalous convection caused by the large-scale curl-free flow, such as equatorial Kelvin wave (e.g. Yang and Ingersoll 2013), is not considered here. The convection, which peaks at $2.3 \tau_{u}$ after the seeding (to make convergence start from close to zero), depends on the spatio-temporal updraft number density $\rho_{u}(\mathbf{x}, t)$ (unit: $\mathrm{m}^{-2} \mathrm{~s}^{-1}$ ):

$$
\rho_{u}=\max \left\{\rho_{u 0}\left(1-\eta \mathrm{E} \frac{\bar{\omega}}{\delta_{0}}\right), 0\right\} .
$$

The maximum operator is used to keep $\rho_{u}$ positive, but the threshold is hardly reached until the very late stage. Here $\rho_{u 0}$ is a constant basic state seeding density which balances the radiative 
cooling:

$$
\rho_{u 0}=\frac{\delta_{0}}{\frac{\Delta h}{H} \pi r_{u}^{2}}
$$

As $\delta_{u}$ is proportional to $\rho_{u}$, they are linked via a rescaling:

$$
\delta_{u}=\rho_{u} \frac{\delta_{0}}{\rho_{u 0}}
$$

The $\eta$ in (9) is a fixed nondimensional feedback parameter that measures the sensitivity of $\rho_{u}$ to the spatially-filtered vorticity (denoted as $\bar{\omega}$ ). Substituting (6), (8), and (9) into (2), we see that the $\delta$ as a random field obeys:

$$
\delta \sim \underbrace{-\eta \mathrm{E} \bar{\omega}+\mathrm{E} \omega}_{\text {simulation }} \approx \underbrace{-(\eta-1) \mathrm{E} \bar{\omega}}_{\text {theory }}
$$

The " $\sim$ " in (12) means $\delta$ as a random variable obeys a distribution, which is the relation used in the numerical simulation. The " $\approx$ " in $(12)$ is a further simplification that assumes the Ekman spin down to work at the filtered scale, which will only be used in the theoretical model in section 3 .

A schematic diagram of this quasi-random seeding scheme is shown in Fig. 2. The general probabilistic relationship between convection and vorticity was noticed by Ritchie and Holland (1997) using observational data. Equation (12) is an update of the classic deterministic relationship between updraft and Ekman pumping flux ("cooperative intensification view”, Ooyama 1969; Schecter and Dunkerton 2009; Montgomery and Smith 2014) to a quasi-stochastic and nonlocal relationship. A larger $\eta$ corresponds to a stronger conditional instability, which is favored by a moister and warmer boundary layer, as well as a moister free troposphere. For $\eta>1$, the convergence induced by deep convection outweighs the Ekman spin down, causing net spin up. Ooyama (1969) let $\eta$ depend on boundary layer equivalent potential temperature, which can be further linked to wind-induced surface heat flux. We use a fixed $\eta$ for simplicity, so the model reduces to the conditional instability of the second kind (CISK) (Montgomery and Smith 2014), but with 
a nonlocal and quasi-random modification. As deep convection is the source of both vapor and vertical vorticity, we consider the $\omega$ in this model to partly represent moisture. Thus, the positive moisture and radiation feedback is implicitly included, and can be considered to modulate $\eta$.

We use a Gaussian filter operator to subtract the mesoscale field, because it only uses neighboring information and yields good math property:

$$
\bar{\omega}(\mathbf{x}, t) \equiv \frac{1}{\pi l^{2}} \iint \exp \left(-\frac{\left|\mathbf{x}-\mathbf{x}^{\prime}\right|^{2}}{l^{2}}\right) \omega\left(\mathbf{x}^{\prime}, t\right) d \mathbf{x}^{\prime}
$$

Here $l$ is a prescribed filter length scale, which represents the nonlocal trigger length scale and is viewed as the mesoscale in this paper. One property is that filtering twice has a stronger smoothing effect than filtering once. Physically, it means the convective region has a tendency to laterally expand due to the nonlocal trigger by cold pool and gravity wave. In appendix A, we show that the system length is a balance between the lateral expansion and the growth in magnitude when the convergent flow is weak $\left(\bar{\omega} \lesssim f_{0}\right)$, and a balance between the lateral expansion and the convergence when the convergent flow is strong $\left(\bar{\omega} \gtrsim f_{0}\right)$. Both balances lead to a flow characteristic length scale of $l$. In addition, the merger tendency of eddies renders an "eddy tension" that makes the scale shrink. It is shown that this effect makes the scale decrease with increasing $-\Delta h / H$. As we have not figured out a method to quantify the "eddy tension", $l$ is regarded as the system length scale throughout the theoretical model in section 3. Note that in the limit of $l \gg L$, the quasi-random scheme reduces to a purely-random scheme.

The problem can be nondimensionalized by choosing $l$ as the length scale and $-\delta_{0}^{-1}$ as the time scale. This leads to five independent nondimensional parameters: $\delta_{0} / f_{0}, r_{u} / l,-\Delta h / H, \eta$ and $\mathrm{E}$. The normalized domain size $L / l$ is another potential parameter especially for the later stage when there are only a few dominant vortices. 


\section{b. The numerical simulation setup}

The barotropic simulation is run in a doubly periodic domain. The numerical code uses Fourier spectral method, and the readers are referred to Fu and O'Neill (2021) for details. The domain size $L$ is chosen to be smaller than Rossby deformation radius $L_{R}$. In the RRCE simulations (e.g. Khairoutdinov and Emanuel 2013), the compensating descent of an updraft is constrained within $L_{R}$ distance from the source in the geostrophic adjustment, so the convection-driven vortices rarely interact with each other when their distances are over $L_{R}$. This is similar to the phenomenon in doubly-periodic domain that a vortex can no longer merge when it is alone.

The reference parameter set is $L=1000 \mathrm{~km}$, grid number $576^{2}, \delta_{0}=-\delta_{\text {rad }}=-7.1 \times 10^{-6}$ $\mathrm{s}^{-1}, \eta=1.6, h_{E}=800 \mathrm{~m},-\Delta h / H=-\delta_{u m} \tau_{u}=8 / 5, H=5 \mathrm{~km}, l=60 \mathrm{~km}, r_{u}=8 \mathrm{~km}, \tau_{u}=$ $2000 \mathrm{~s}$, and $f_{0}=10^{-4} \mathrm{~s}^{-1}$ (equivalent to $43^{\circ} \mathrm{N}$ ). To guarantee $\rho_{u}$ is positive in (9), it requires $\bar{\omega} / f_{0}>-(\eta \mathrm{E})^{-1}\left(-\delta_{0} / f_{0}\right) \approx-0.55$. An artificial viscosity of $200 \mathrm{~m}^{2} \mathrm{~s}^{-1}$ is added to dissipate the grid-scale enstrophy.

Using $\Delta \theta=20 \mathrm{~K}$ which makes the atmospheric lapse rate close to moist adiabatic, the $\delta_{0}$ is equivalent to a radiative cooling rate of $Q_{\text {rad }} \sim \delta_{0} \Delta \theta \sim-12.3 \mathrm{~K} \mathrm{day}^{-1}$. This is much larger than the typical tropical value of $-1 \sim-2 \mathrm{~K} \mathrm{day}^{-1}$ (Hartmann et al. 2001). The motivation of using such a high-magnitude $\delta_{0}$ and $f_{0}$ is to save computational resource by accelerating the spin up. The key nondimensional parameter values for the reference test are: $-\delta_{0} / f_{0}=0.07$ (a large $-\delta_{0}$ and large $f_{0}$ yields a moderate $\left.-\delta_{0} / f_{0}\right), r_{u} / l=0.133, \Delta h / H=-1.6, \mathrm{E}=0.08, \eta=1.6$. In the simulations, we fix $L$ and $r_{u}$, but change $l, \eta,-\Delta h / H$, and $f_{0}$, one at a time. We pay more attention to $l$ which is conceptually the most important one to this quasi-random model. The $l=60 \mathrm{~km}$ test is designated as the reference test, and the $l=30 \mathrm{~km}$ and $l=45 \mathrm{~km}$ tests are the sensitivity tests. We encourage the readers to watch the movie of the simulation in the supplemental material. 
c. The numerical simulation result

First, we check the $l=60 \mathrm{~km}$ test. The $\omega$ field at six snapshots is shown in Fig. 3. Introducing the nondimensional time coordinate $t^{\prime}=-\delta_{0} t$, the snapshots are at $t_{1}^{\prime}=0.55, t_{2}^{\prime}=1.54, t_{3}^{\prime}=2.54$, $t_{4}^{\prime}=3.54, t_{5}^{\prime}=4.54$ and $t_{6}^{\prime}=5.54$. At $t_{1}^{\prime}$, there are sporadic concentrated vorticity patches. At $t_{2}^{\prime}$, there are more and more high vorticity patches which are produced by the "lucky" repetitive occurrence of convective events on the existing patches. These high vorticity patches become nuclei that collect other small vortices on their way, and merge with each other. At $t_{3}^{\prime}$, the patches with the most concentrated vorticity began to grow in a positive feedback. Loosely-organized vortex clusters that consist of multiple vorticity patches appear. As the convective seeding depends on vorticity, a vortex cluster is also a convective cluster. The vortex cluster is intensified via repetitive vorticity stretching by the dense updrafts. This process corresponds to the "vortical hot tower" route of tropical cyclogenesis in the real atmosphere (Hendricks et al. 2004; Montgomery et al. 2006; Kilroy et al. 2017). The clusters become more compact and axisymmetric due to the convergent flow, the merger of small vortices within the cluster, and the adjustment by vortex Rossby wave. The clusters also merge with each other, as is more clearly seen from the $\bar{\omega}$ field (Fig. 4). Such a multiscale merger process has been reported in cloud-permitting simulations (Ritchie and Holland 1997; Hendricks et al. 2004; Fang and Zhang 2011; Schecter 2016, 2017), and reminds us of the galaxy merger process (e.g. Mihos and Hernquist 1996). Finally, a single dominant vortex cluster is left in the domain. Meanwhile, other regions have less and less convection due to the growth of negative vorticity, which is the other side of this positive feedback.

Then, we analyze the sensitivity test: the pure random seeding test, the $l=30 \mathrm{~km}$ test and the $l=45 \mathrm{~km}$ test. Figures 5 and 6 show that the vorticity field of all tests at $t_{1}^{\prime}$ looks similar, with smaller $l$ leading to larger $\bar{\omega}$ magnitude and more clusters. As the mesoscale feedback and vortex 
motion is not vigorous yet, this is largely a stochastic result. The later stage evolution is quite different:

- The purely random test remains at the sporadic vorticity stage, with $\bar{\omega}$ fluctuating around a quasi-equilibrium state. The Ekman spin down is strong enough to suppress any strong vorticity patch (produced by chance) before it grows into a cluster by merger.

- When the positive feedback is included, a smaller $l$ leads to faster local intensification due to the higher $\bar{\omega}$ provided by the purely random stage. However, the further merger is less prominent due to the vortex clusters' relatively small size ("collision cross-section"), and the system resembles a point vortex system.

As the vortex cluster size seems to scale as $l$, we track the highest $\bar{\omega}$ point and calculate the radial structure of the azimuthally-averaged $\omega$ (Fig. 7). The shape is confirmed to be similar, with $l$ as the length scale, in agreement with our theoretical prediction in appendix A. The saturation in scale is different from the free-decaying $2 \mathrm{D}$ turbulence where the vortex size keeps growing via merger (e.g. McWilliams 1990). This scale-invariant property in merger will be further discussed in section $3 \mathrm{c}$ and theoretically explained to be mainly due to the convergent flow in appendix A.

Figure 8a shows the number of local maximum $\bar{\omega}$ points in the domain, $N$, which represents the number of vortex clusters. Near the beginning, there is $N \sim l^{-2}$. In section $3 b$ it will be shown to be the result of Gaussian filter on a random field. In the subsequent evolution, $N$ drops significantly due to merger. As the number of merger events experienced by each vortex is quite different, the peak $\bar{\omega}$ magnitude of the vortex clusters become more widespread (e.g. Fig. 4). This reminds us of the droplet spectrum widening via stochastic collision-coalescence in cloud microphysics (e.g. Yau and Rogers 1996). 
Finally, we check the vorticity standard deviation $\operatorname{std}(\omega)$ (Fig. 8b). For all tests, it grows rapidly within $t^{\prime} \lesssim 0.5$. The $\operatorname{std}(\omega)$ of the purely random test reaches an equilibrium since $t^{\prime} \sim 0.5$, and the other tests keep growing slowly. Such a growth rate transition, as well as the separation of the purely random test from those quasi-random tests, indicate a clear transition from a stochastic regime to a deterministic regime by $t^{\prime} \sim 0.5$.

\section{Theory}

\section{a. The basic idea}

Based on the analysis in section $2 \mathrm{c}$, we build a theoretical model that separately treats the stochastic regime and the deterministic regime. In the stochastic regime, the vorticity grows due to random stretching of the randomly seeded convection against the radiative cooling and Ekman spin down. A purely statistical description is sufficient. In the deterministic regime, we model the mean vorticity of the vortex cluster (close to but not the same as $\bar{\omega}$ ), which is a mesoscale property. It takes the filtered end state value of the statistical regime model as its initial condition.

\section{b. The stochastic regime}

The movie of $\bar{\omega}$ evolution shows that the first round of vortex clusters produced by random stretching does not vanish. They grow steadily according to the (positive) physical feedback. Thus, we use the theoretically predicted equilibrium state of the stochastic regime as the initial condition of the deterministic regime model. There is a prerequisite for the regime separation: when the equilibrium is just reached, the preferential seeding signal driven by the feedback should be much smaller than the stochastic component. In appendix B, a criterion based on signal-to-noise ratio is presented, and the separation is shown to be roughly valid for the reference test. 
Because a free-evolving randomly forced field gradually feels the damping and reaches the vorticity quasi-equilibrium (VQE) in a damping time scale $T_{0}$, we estimate the equilibrium field as a field that evolves freely to $t=T_{0}$. The $T_{0}$ is estimated with the Ekman spin down rate plus the radiative cooling rate:

$$
T_{0}=\left(f_{0} \mathrm{E}-\delta_{0}\right)^{-1}
$$

Here, we have assumed that $\bar{\omega} \ll f_{0}$, as is in most of our cases. This makes the classic linear Ekman spin down time scale $\left(f_{0} \mathrm{E}\right)^{-1}$ available. Equation (14) predicts $-\delta_{0} T_{0}=0.47$ for the reference test, which agrees with the transition time in Fig. 8b. We estimate the characteristic amplitude of the randomly-forced field with the Fourier spectrum of $\omega$.

First, we show that if it is the filtered field rather than the original field that is of interest, the random stretching process which involves convergence can be approximated as a geometrical random superposition of fixed increments. This is because, if the area of an isolated vorticity patch $S$ (surrounded by near-zero $\omega$ ) is much smaller than $l^{2}$, only its circulation change $\Delta \Gamma$ produced by seeding a convection will influence the filtered value:

$$
\Delta \Gamma=-f_{0} \pi r_{u}^{2} \frac{\Delta h}{H}
$$

The $\Delta \Gamma$ is proportional to the total mass sink in an updraft event. In contrast, the local vorticity magnitude evolution in the updraft region grows more steeply. The local relative vorticity obeys a power law due to the conservation of absolute circulation ( Fu and O'Neill 2021): $\omega_{m}=f_{0}(1-$ $\Delta h / H)^{m}-f_{0}$, where $m$ is the total number of updrafts falling to one place. To make the discussion more general, we use a scalar $\phi$ to denote $\omega$. This problem transforms to studying the filtered field of the random superposition of $N_{u}$ Gaussian shape increments, denoted as $\phi_{n}$ :

$$
\phi_{n}=\Phi_{0} \exp \left[-\frac{\left(x-x_{n}\right)^{2}+\left(y-y_{n}\right)^{2}}{r_{u}^{2}}\right], \quad n=1,2, \ldots, N_{u}
$$


whose length scale is $r_{u}$ and the peak increment $\Phi_{0}$ is:

$$
\Phi_{0}=-f_{0} \frac{\Delta h}{H}
$$

The $N_{u}$ is the total number of updrafts within $T_{0}$ time inside the domain, which is estimated with (10):

$$
N_{u}=\rho_{u 0} L^{2} T_{0}=\frac{-\delta_{0} T_{0}}{-\frac{\Delta h}{H} \frac{\pi r_{u}^{2}}{L^{2}}} .
$$

The finite-domain Fourier transform of $\phi_{n}$ is denoted as $\hat{\phi}_{n}$ :

$$
\begin{aligned}
\hat{\phi}_{n} & \equiv \frac{1}{L^{2}} \int_{-L / 2}^{L / 2} \int_{-L / 2}^{L / 2} \phi_{n} \exp \left[-i\left(k_{x} x+k_{y} y\right)\right] d x d y \\
& \approx \gamma \Phi_{0} \frac{r_{u}^{2}}{4 \pi} \exp \left[-\frac{r_{u}^{2}\left(k_{x}^{2}+k_{y}^{2}\right)}{4}\right] \underbrace{\exp \left[i\left(k_{x} x_{n}+k_{y} y_{n}\right)\right]}_{\text {random shift factor }},
\end{aligned}
$$

for wavenumbers:

$$
k_{x}=\frac{2 \pi}{L} m_{x}, k_{y}=\frac{2 \pi}{L} m_{y}, \quad m_{x}, m_{y} \in\left\{-\frac{N}{2}, \ldots 0, \ldots, \frac{N}{2}-1\right\} .
$$

In (19), we have used the infinite-domain Fourier transform of Gaussian function to approximate the finite-domain transform, which is valid due to $r_{u} \ll L$. The parameter $\gamma=4 \pi^{2} / L^{2}$ in (19) is a conversion coefficient.

Second, we show that the power spectrum of the randomly superposed field $\phi=\sum_{n=1}^{N_{u}} \phi_{n}(x, y)$, $|\hat{\phi}|$, is proportional to the individual one $\left|\hat{\phi}_{n}\right|$ :

$$
\begin{aligned}
|\hat{\phi}| & =\gamma \Phi_{0} \frac{r_{u}^{2}}{4 \pi} \exp \left[-\frac{r_{u}^{2}\left(k_{x}^{2}+k_{y}^{2}\right)}{4}\right]\left|\sum_{n=1}^{N_{u}} \exp \left[i\left(k_{x} x_{n}+k_{y} y_{n}\right)\right]\right| \\
& \approx \underbrace{\gamma \Phi_{0} \frac{r_{u}^{2}}{4 \pi} \exp \left[-\frac{r_{u}^{2}\left(k_{x}^{2}+k_{y}^{2}\right)}{4}\right]}_{\left|\hat{\phi}_{n}\right|} N_{u}^{1 / 2} .
\end{aligned}
$$

The modulus of the sum of the random shift factor, which is the amplitude of a group of incoherent waves, is $N_{u}^{1 / 2}$ (e.g. Weisstein 2021), which is proportional to $T_{0}^{1 / 2}$. This scaling generally agrees with the spectrum of the simulation (Fig. 9a), though the spectrum shape has some deviation due 
to the presence of damping and the convergent flow. The proportional coefficient $N_{u}^{1 / 2}$ is not $N_{u}$, which instead represents the superposition of coherent waves that originates from the stationary growth of an existing pattern without damping.

Third, we use $|\hat{\phi}|$ to estimate the characteristic amplitude of $\bar{\phi}$ (the spatially filtered $\phi$ ). As the filter is a convolution in physical space, the modulus of the filtered field $|\hat{\bar{\phi}}|$ is obtained by multiplying $\exp \left[-l^{2}\left(k_{x}^{2}+k_{y}^{2}\right) / 4\right]$ to $\hat{\phi}$ and taking the modulus, using (21):

$$
\begin{aligned}
|\hat{\bar{\phi}}| & =\gamma \Phi_{0} N_{u}^{1 / 2} \frac{r_{u}^{2}}{4 \pi} \exp \left[-\frac{1}{4}\left(r_{u}^{2}+l^{2}\right)\left(k_{x}^{2}+k_{y}^{2}\right)\right] \\
& \approx \gamma \Phi_{0} N_{u}^{1 / 2} \frac{r_{u}^{2}}{4 \pi} \exp \left[-\frac{1}{4} l^{2}\left(k_{x}^{2}+k_{y}^{2}\right)\right] .
\end{aligned}
$$

Here we have used $r_{u}^{2} \ll l^{2}$ to simplify the expression. The filter roughly truncates the lowest significant wavenumber to $2 / l$. The corresponding cutoff wavelength is $2 \pi /(2 / l)=\pi l$. As the filter significantly damps the Fourier modes whose wavelength is shorter than $l$, the number of $\bar{\phi}$ peaks in the domain (denoted as $N_{l}$ ) should obey:

$$
N_{l}=\left(\frac{L}{\pi l}\right)^{2}
$$

which is in good agreement with the simulation (Fig. 9b). Suppose the $\bar{\phi}$ field consists of $N_{l}$ randomly distributed Gaussian-shape structures, with a length scale of $l$ and an amplitude scale of $\Phi_{l}$. By mimicking (22), we get:

$$
|\hat{\bar{\phi}}| \approx \gamma \Phi_{l} N_{l}^{1 / 2} \frac{l^{2}}{4 \pi} \exp \left[-\frac{1}{4} l^{2}\left(k_{x}^{2}+k_{y}^{2}\right)\right] .
$$


Equating (22) and (24), and considering that the vortex cluster amplitude $\omega_{0}$ is a fixed $\lambda=2$ multiple of $\Phi_{l}$, we get :

$$
\begin{aligned}
\omega_{0} & =\lambda \Phi_{l} \\
& =\lambda\left(\frac{N_{u}}{N_{l}}\right)^{1 / 2} \frac{r_{u}^{2}}{l^{2}} \Phi_{0} \\
& =f_{0} \lambda\left(\delta_{0} T_{0} \frac{\Delta h}{H} \frac{r_{u}^{2}}{\pi l^{2}}\right)^{1 / 2},
\end{aligned}
$$

where we have substituted in (17), (18) and (23). The $\lambda$ is a tuning factor that calibrates our coarse theory to the simulation. Figure $9 \mathrm{c}$ shows that (25) generally agrees with the simulation where $\omega_{0}$ is compared with the strongest vortex cluster, though the sensitivity to $l$ is underestimated for the reference case $(-\Delta h / H=1.6)$. The underestimation is likely due to the heterogeneity of vortex cluster strength. A potential future work is to model the vorticity probability distribution function with a Markov chain (e.g. Fu and O’Neill 2021), and use it to inform the distribution of vortex cluster strength.

Transforming back from $\phi$ to $\omega$, we summarize that the stochastic regime model provides the initial condition for the deterministic regime model which is a dynamical system of three variables: the vortex cluster radius $a$, the spatial cluster number density $N_{v}$ (unit: $\mathrm{m}^{-2}$, rather than $\mathrm{m}^{-2} \mathrm{~s}^{-1}$ as is for $\rho_{u}$ ), and the relative circulation $\Gamma$ of each cluster. Their initial conditions are denoted as $a_{0}, N_{v 0}$ and $\Gamma_{0}$ respectively:

$$
\left.a_{0} \equiv a\right|_{t=T_{0}}=l,\left.\quad N_{v 0} \equiv N_{v}\right|_{t=T_{0}}=\left(\pi a_{0}\right)^{-2},\left.\quad \Gamma_{0} \equiv \Gamma\right|_{t=T_{0}}=\pi a_{0}^{2} \omega_{0}
$$

\section{c. The deterministic regime}

Though it is always stochastic at the convective scale, the mesoscale is more deterministic due to the filter which makes the convective probability smoother in space. A smoother and larger convective number density $\rho_{u}$ (probability) makes the random field more closely obey the mesoscale 
feedback. We assume the mesoscale dynamics is completely deterministic after the vorticity quasiequilibrium is established in the stochastic regime. In appendix $\mathrm{C}$, we quantify the degree of determinacy (fluctuation) by defining and calculating a "convective Knudsen number".

The nonlinear terms in the governing equations (1)-(4) make the system chaotic, so we only solve the vortex cluster's characteristic relative circulation $\Gamma$ and the spatial number density $N_{v}$. The idea is to adapt the vortex gas model in studying two dimensional turbulence (Carnevale et al. 1991; Trizac 1998) to the tropical mesoscale vortex cluster. The vortex clusters are treated as identical particles that undergo random collision-coalescence. As a strong approximation, the heterogeneity of size and strength are neglected, which is more valid for the early stage. There are two major differences from the classic vortex gas model.

- First, we study the merger of vortex cluster which is a loose complex of multiple small vortices and filaments accompanied by a strong central vortex. This is different from the vortex gas model where one particle denotes one vortex. Until the late stage, the central vortex in a cluster only takes a small fractional area within $l$ radius, so the filaments produced by the merger of the central vortex are still within $l$ radius, and therefore $\Gamma$ is generally conserved.

- Second, there is convergent/divergent flow. This is close to the point vortex-sink system proposed by Novikov and Novikov (1996), where the streamline associated with each vortex is helical. Many previous studies have found that the updraft-induced convergent flow favors the merger of convective vortices (Hendricks et al. 2004; Fang and Zhang 2011; Schecter 2016, 2017).

The first point is further explained here. Within a vortex cluster of radius $l$, the central vortex radius (denoted as $r_{c}$ ) is basically determined by the competition between the expansion due to the filter, against the contraction due to convergence and merger. The initial value of $r_{c}$ should 
scale as $r_{u}$, so $r_{c} \ll l$. In the classic vortex-gas model, the merger between two vortices with identical vorticity yields $\Gamma_{3}^{2}=\Gamma_{1}^{2}+\Gamma_{2}^{2}$ and $a_{3}^{4}=a_{1}^{4}+a_{2}^{4}$, where the subscript 1 and 2 denote the two vortices before merger and 3 denotes the merged vortex (Carnevale et al. 1991; Trizac 1998). The $\Gamma^{2}$ conservation is obtained from the conservation of kinetic energy: $\Gamma^{2} \sim \omega^{2} r_{c}^{4}$ (unit: $\mathrm{m}^{4}$ $\left.\mathrm{s}^{-2}\right)$. As $\omega$ is a tracer, the $\Gamma^{2}$ conservation readily leads to the fourth order conservation of $r_{c}$. The net loss of circulation represents the filaments produced in the mutual shearing process of a merger event. As we look at the vortex cluster scale, the $r_{c} \ll l$ property and the convergent flow make the filaments well contained within a radius of $l$, and keep the central vortex small. Thus, the circulation $\Gamma$, rather than $\Gamma^{2}$, should be conserved. In Appendix A, we show that the cluster radius is generally constrained to be no larger than $l$ by the convergent flow, so we will use $a=l$.

To apply the vortex gas idea to vortex clusters, we let $\langle\omega\rangle$ denote the mesoscale $\omega$ characteristic magnitude of vortex clusters at time $t$, and then model its evolution. Our vortex gas model assumes equal strength of all vortex clusters at any time. In the theoretical model, a vortex cluster is considered to be a top-hat structure with a radius of $l$, with the $\Gamma$ being linked to $\langle\omega\rangle$ via:

$$
\langle\omega\rangle \equiv \frac{\Gamma}{\pi a^{2}}=\frac{\Gamma}{\pi l^{2}} \quad \text { (theory). }
$$

The \langle\rangle is an operator that can also be performed on other variables. In the simulation, however, the magnitude of the clusters varies significantly. Among them, the strongest cluster best represents the final major vortex. Thus, in processing the simulation data, we define $\langle\omega\rangle$ as a conditional average within $2 l$ radius, centralizing at the maximum $\bar{\omega}$ point $\left(\mathbf{x}_{\mathbf{c}}\right)$ :

$$
\langle\omega\rangle \equiv \frac{1}{\pi(2 l)^{2}} \iint_{\left|\mathbf{x}-\mathbf{x}_{\mathbf{c}}\right|<2 l} \omega d \mathbf{x} \quad \text { (diagnosis). }
$$

The $2 l$ radius is chosen to encompass most of the positive $\omega$ within the vortex cluster (e.g. Fig. 7). The $\langle\omega\rangle$ defined in (27) corresponds to a more compact vortex cluster structure than that in (28), but the former represents the average strength and the latter represents the strongest vortex 
cluster. As a compromise, we consider them to be comparable in this preliminary investigation. In the future, a more careful comparison can be made when the heterogeneous strength of the vortex clusters are considered.

We need to estimate the magnitude ratio of the convergent to rotational wind to determine whether the convergent flow is important. From (2), the net convergence $\delta_{n}$ is:

$$
\delta_{n}=\left\langle\delta_{u}\right\rangle-\delta_{0}+\left\langle\delta_{E}\right\rangle=-(\eta-1) \mathrm{E}\langle\omega\rangle,
$$

which yields $-\delta_{n} /\langle\omega\rangle=(\eta-1) \mathrm{E}=0.048$ for the reference test. As this value is much smaller than one, the effect of convergent flow in driving the motion of the vortex clusters is ignored.

The time scale of merger $\tau_{m}$ is estimated with the Boltzmann mean collision time (Trizac 1998):

$$
\tau_{m}=\frac{\alpha_{m}}{N_{v} a \Gamma / r}=\frac{\alpha_{m}}{\pi l^{3}} N_{v}^{-3 / 2}\langle\omega\rangle^{-1},
$$

where $r$ is the characteristic distance between two vortices that obeys $r \sim N_{v}^{-1 / 2}$, and $\Gamma / r$ is the characteristic drifting velocity. The $\alpha_{m}$ is a coefficient near unity, which we subjectively choose as a fixed 1.6 to fit the simulation result. Equation (30) leads to the governing equation of $N_{v}$ :

$$
\frac{d N_{v}}{d t}=-\frac{N_{v}}{\tau_{m}}
$$

Equation (27) shows that the evolution of $\langle\omega\rangle$ is equivalent to that of $\Gamma$, due to $a=l$. Applying the \langle\rangle in theoretical sense to (1), we get:

$$
\begin{aligned}
\frac{d\langle\omega\rangle}{d t} & \approx \frac{\langle\omega\rangle}{\tau_{m}}-\delta_{n}\left(\omega^{-}+f_{0}\right) \\
& =\underbrace{\frac{\langle\omega\rangle}{\tau_{m}}}_{\text {merger }}+\underbrace{\frac{\langle\omega\rangle}{\tau_{e}}-\delta_{n} \omega^{-}}_{\text {entrain }},
\end{aligned}
$$

where we have used Gauss theorem, and the loop integral encapsulates most of the positive $\omega$ region. The $\omega^{-}$is the "environmental relative vorticity", which is also entrained into the vortex cluster. When diagnosed from simulation, it is defined as the average vorticity outside of the 
vorticity clusters (the area excluding all $2 l$-radius patches). The $\tau_{e}$ is the timescale of growth purely due to entraining environmental planetary vorticity:

$$
\tau_{e} \equiv\left[(\eta-1) \mathrm{E} f_{0}\right]^{-1}
$$

Substituting in the reference parameter, we get $-\delta_{0} \tau_{e} \approx 1.5$. When the vortex is not involved in a merger event, the stream function contours are closed and roughly perpendicular to the vorticity gradient vector, so the rotational wind $\mathbf{u}_{\omega}$ does not induce net transport of vorticity to the vortex. During the episodic merger events, the contour lines are open, and the role of $\mathbf{u}_{\omega}$ is parameterized as the merger term $\langle\omega\rangle / \tau_{m}$.

We need to solve $\omega^{-}$to make (31) and (32) a closed dynamical system. The $\omega^{-}$becomes more and more negative due to the radiative cooling and Ekman suction. Figure 10 shows that by $t^{\prime}=3$, the $\omega^{-} / f_{0}$ of the $l=30 \mathrm{~km}$ simulation has dropped to -0.2 . If the environmental absolute vorticity is exhausted $\left(\omega^{-} \rightarrow-f_{0}\right)$, the merger will be the only way to raise vorticity magnitude. This consideration is important at the later stage of the evolution. One way to estimate $\omega^{-}$is using the domain-integrated conservation of absolute vorticity: a faster growth of the cyclonic vortex patch leads to a faster consumption of the environmental vorticity:

$$
\omega^{-}=-\langle\omega\rangle \frac{N_{v} \pi l^{2}}{1-N_{v} \pi l^{2}} \approx-\langle\omega\rangle N_{v} \pi l^{2} .
$$

Here we have used an approximation that the cyclonic vorticity patches only take a relatively small fractional area at the beginning $\left(N_{v 0} \pi l^{2}=1 / \pi\right)$, and it further drops due to merger. Substituting (29) into (32), we get:

$$
\frac{d \ln \langle\omega\rangle}{d t}=\frac{1}{\tau_{m}}+(\eta-1) \mathrm{E}\left(\omega^{-}+f_{0}\right) .
$$


Note that $\tau_{m}$ is also a function of $\langle\omega\rangle$. Substituting (31) and (35) into (34), we find that $-\omega^{-}$ grows a bit slower than exponentially:

$$
\begin{aligned}
\frac{d \ln \left(-\omega^{-}\right)}{d t} & \approx \frac{d \ln \langle\omega\rangle}{d t}+\frac{d \ln N_{v}}{d t} \\
& =\frac{1}{\tau_{m}}+(\eta-1) \mathrm{E}\left(\omega^{-}+f_{0}\right)-\frac{1}{\tau_{m}} \\
& =(\eta-1) \mathrm{E}\left(\omega^{-}+f_{0}\right) .
\end{aligned}
$$

The cancellation of $1 / \tau_{m}$ term indicates that the merger does not influence $\omega^{-}$. This is because the total circulation of the vortices are conserved during merger: the rise of $\langle\omega\rangle$ is exactly cancelled by the decrease of the total area of the vortex clusters. An initial condition of $\omega^{-}$is required to solve (36). It is estimated with $\left.\langle\omega\rangle\right|_{t=T_{0}}=\omega_{0}$ (25), and the cyclonic patch's initial fractional area from $\left.N_{v}\right|_{t=T_{0}}=N_{\nu 0}=(\pi l)^{-2}(26)$ :

$$
\left.\omega^{-}\right|_{t=T_{0}}=-\omega_{0} \frac{N_{\nu 0} \pi l^{2}}{1-N_{\nu 0} \pi l^{2}}=-\omega_{0} \frac{1}{\pi-1} .
$$

Equations (36) and (37) yield an approximate analytical solution of $\omega^{-}$as a function of time:

$$
\omega^{-}(t) \approx-\frac{\omega_{0}(\pi-1)^{-1}}{1+\frac{\omega_{0}}{f_{0}}(\pi-1)^{-1} \exp \left(\frac{t}{\tau_{e}}\right)} \exp \left(\frac{t}{\tau_{e}}\right)
$$

In deriving (38), we have used $f_{0}+\omega_{0} \pi^{-1} \approx f_{0}$, because $\omega_{0} \pi^{-1}<\omega_{0} \ll f_{0}$.

The governing equation of $N_{v}$ (31) and $\langle\omega\rangle$ (32), as well as the approximate expression of $\omega^{-}$(38) and the initial condition (26) render a nonlinear ordinary differential equation (ODE) system which does not have an exact analytical solution. If we let $\omega^{-} \approx 0$ in (32), an approximate analytical solution can be obtained, which is plotted in Fig. 11. The technique is to find the first integral $\langle\omega\rangle N_{v} \sim \exp \left[\left(t-T_{0}\right) / \tau_{e}\right]$ which represents the total circulation of the vortex cluster region. We have:

$$
\langle\omega\rangle \approx \omega_{0}\left\{1+\frac{\exp \left[\left(t-T_{0}\right) / \tau_{e}\right]-1}{2 \tau_{m 0} / \tau_{e}}\right\}^{2} \exp \left(\frac{t-T_{0}}{\tau_{e}}\right)
$$




$$
N_{v} \approx N_{v 0}\left\{1+\frac{\exp \left[\left(t-T_{0}\right) / \tau_{e}\right]-1}{2 \tau_{m 0} / \tau_{e}}\right\}^{-2}
$$

459

$$
\tau_{m} \approx \tau_{m 0}\left\{1+\frac{\exp \left[\left(t-T_{0}\right) / \tau_{e}\right]-1}{2 \tau_{m 0} / \tau_{e}}\right\} \exp \left(-\frac{t-T_{0}}{\tau_{e}}\right)
$$

where $\tau_{m 0}$ is the initial value of $\tau_{m}$. Using (26), we see it is inversely proportional to $\omega_{0}$ :

$$
\tau_{m 0}=\left.\tau_{m}\right|_{t=T_{0}}=\frac{\alpha_{m}}{\pi l^{3}} N_{\nu 0}^{-3 / 2} \omega_{0}^{-1}=\pi^{2} \alpha_{m} \omega_{0}^{-1}
$$

The $\tau_{m 0}$ does not directly depend on $l$. This is because a smaller $l$ leads to a shorter vortex interval which enhances mutual advection and shortens the collision path, but the collision cross-section is also smaller. The $N_{v}$ is predicted to decrease with time due to merger. The fractional decrease $\left(\left.N_{v}\right|_{t=T_{0}}-N_{v}\right) /\left.N_{v}\right|_{t=T_{0}}$ is larger for a smaller $l$ test which has a larger $\omega_{0}$ (Fig. 11b). Physically, it is because the smaller initial vortex interval and the subsequent more vigorous vortex motion outweighs the disadvantage of smaller collision cross-section $l$.

The reference parameter yields $\tau_{m 0} / \tau_{e}=5.8$, so merger is not vigorous at the beginning of the deterministic regime, and we can well assume $\tau_{m 0} / \tau_{e}>1$ for any case of interest. In general, at the small $t$ regime $\left(t / \tau_{e} \ll \ln \left(2 \tau_{m 0} / \tau_{e}\right)\right)$, the asymptotic expressions of $\langle\omega\rangle, N_{v}$ and $\tau_{m}$ are:

$$
\langle\omega\rangle \sim \omega_{0} \exp \left(\frac{t-T_{0}}{\tau_{e}}\right)
$$

$$
N_{v} \sim N_{v 0},
$$

$$
\tau_{m} \sim \tau_{m 0} \exp \left(-\frac{t-T_{0}}{\tau_{e}}\right)
$$

They indicate that the growth is solely due to entrainment, and the flow pattern is similar to a linear instability growth at a preferred wavelength. For large $t\left(t / \tau_{e} \gg \ln \left(2 \tau_{m 0} / \tau_{e}\right)\right)$, the merger rate, which is a nonlinear upscale growth factor, outweighs entrainment and asymptotically reaches 
twice its magnitude:

$$
\begin{gathered}
\langle\omega\rangle \sim \omega_{0}\left(\frac{\tau_{e}}{2 \tau_{m 0}}\right)^{2} \exp \left(\frac{t-T_{0}}{\tau_{e} / 3}\right), \\
N_{v} \sim N_{v 0}\left(\frac{\tau_{e}}{2 \tau_{m 0}}\right)^{-2} \exp \left(-\frac{t-T_{0}}{\tau_{e} / 2}\right), \\
\tau_{m} \sim \frac{\tau_{e}}{2} .
\end{gathered}
$$

The flow pattern becomes chaotic. In this large $t$ regime, the vortices are far away from each other, and the reason for keeping a steady merger rate comes from the increasing $\langle\omega\rangle$ due to entrainment. If the environmental negative relative vorticity $\omega^{-}$is considered, entrainment will become less efficient, and the growth due to merger will become less and less effective as well. The general evolution picture reminds us of rotating Rayleigh-Bénard convection. As the lower and upper lid temperature becomes larger, the flow transits from the laminar vortex grid regime to the turbulent single vortex regime, which is also a poorly understood process (Stellmach et al. 2014; Guervilly et al. 2014; Favier et al. 2014).

Figure 11 compares the analytical solution of $\langle\omega\rangle, N_{v}$ and $\tau_{m}$ with the numerical solution of the ODEs ((31), (32) and (38)) that consider finite $\omega^{-}$. The deviation becomes discernible only at the later stage where $\left|\omega^{-} / f_{0}\right| \ll 1$ is no longer valid.

\section{Comparison with barotropic simulation}

In this section, the numerical integration of the ODEs is compared to the barotropic simulation. Figure 10 shows the time series of $\langle\omega\rangle, \omega^{-}$, and an estimated maximum wind $V_{m}$, for tests with different $l, \eta, \Delta h / H$ and $f_{0}$. Note that $f_{0}$ should also implicitly influence $\mathrm{E}$ and $\eta$, but we let them be fixed while changing $f_{0}$. When $\Delta h / H$ is changed, $\tau_{u}$ is changed proportionally to keep the updraft-induced convergence $-\Delta h /\left(\tau_{u} H\right)$ fixed. 
The ODE system successfully predicts the basic trend of the evolution of $\langle\omega\rangle / f_{0}$ : it is larger for a smaller $l$, a larger $\eta$, a larger $-\Delta h / H$ and a larger $f_{0}$. As is predicted by (46), the two parameters that only influence $\omega_{0}: l$ and $-\Delta h / H$, do not significantly change the slope in the log coordinate plotting of $\langle\omega\rangle / f_{0}$ in Fig. 10. Figure 11 shows that the slope is gentler than the prediction of the asymptotic solution (46), due to the entrainment of negative environmental relative vorticity. A larger $\eta$ only accelerates the growth rate in the deterministic regime; a larger $f_{0}$ is predicted to reduce $\omega_{0}$ in the stochastic regime by reducing the equilibrium time $T_{0}$ (hardly justifiable from Fig. 10), but accelerate the deterministic regime by shortening $\tau_{e}$. One significant drawback of our theory is underestimating the sensitivity of $\langle\omega\rangle / f_{0}$ to $l$, which originates from underestimating the sensitivity of $\omega_{0}$ to $l$ (e.g. Fig. 9c).

The prediction of $\omega^{-} / f_{0}$ is good at the early stage, but its magnitude is overestimated at the later stage. The barotropic simulation shows that $\omega^{-} / f_{0}$ is insensitive to $f_{0}$, but we predict $\left|\omega^{-} / f_{0}\right|$ to increase with $f_{0}$. The reason of the deviation has not been figured out.

The maximum wind $V_{m}$ is estimated with the characteristic mesoscale rotational wind $\langle\omega\rangle l$, plus the characteristic cloud-scale convergent wind induced by an individual updraft $V_{u p}$ :

$$
V_{m}=\langle\omega\rangle l+V_{u p} \quad \text { where } \quad V_{u p}=-\frac{1}{\tau_{u}} \frac{\Delta h}{H} \frac{r_{u}}{2} .
$$

The mesoscale convergent wind is negligible, as is shown in (29). A direct summation is used, because the mesoscale rotational wind is smooth in space, and the cloud-scale convergent wind around an updraft traverses all directions. We consider the maximum wind to be attained where the two velocity vectors are parallel. The reference test yields $V_{u p}=3.2 \mathrm{~m} \mathrm{~s}^{-1}$, which is only dominant in (49) at the early stage. The trend of $V_{m}$ generally agrees with the barotropic simulation. However, our theory is still too coarse to quantitatively predict $V_{m}$, because the role of eddy merger and axisymmetrization in concentrating vorticity and therefore accelerating the flow is neglected. 
The eddy acceleration factor proposed by Fu and O'Neill (2021), which links the mean vorticity to the maximum wind, could be combined with (49) in the future.

The $N_{v}$ diagnosed from the simulation (Fig. 8a) qualitatively agrees with the theory at the $t^{\prime} \lesssim 3$ early stage (Fig. 11a), where the size and strength of the vortex clusters do not vary significantly. We do not attempt to fully benchmark $N_{v}$ before a more complete theoretical model with heterogeneous vortex clusters is established.

\section{Cloud-permitting simulation}

\section{a. An overview}

We apply the theory to understand a case of simulated spontaneous tropical cyclogenesis in a cloud-permitting model, which is closer to the real atmosphere. The setup is identical to that reported in Fu and O'Neill (2021), where the intensification of the major vortex is studied. In this section, we focus on the formation of the major vortex from quasi-random convection. It is a full-physics simulation of Cloud Model 1 (Bryan and Fritsch 2002), with $1080 \times 1080 \mathrm{~km}^{2}$ doubly periodic domain, $576 \times 576 \times 65$ grid points, and a horizontal grid interval of $2 \mathrm{~km}$. The sea surface temperature is fixed to $305 \mathrm{~K}$, and the Coriolis parameter is $f_{0}=10^{-4} \mathrm{~s}^{-1}$. The initial sounding is taken from the horizontal-average of a $120 \times 120 \mathrm{~km}^{2}$ small-domain non-rotating simulation running to 100 days, so it is in radiative-convective equilibrium. We refer the readers to $\mathrm{Fu}$ and O'Neill (2021) for more details. The low-mid level vertical relative vorticity, which is defined as the vertical average within the 1.18-6.25 km height level (without density weighting), is denoted as $\omega$.

The domain-averaged rainfall rate in Fig. 12c shows that the system enters a convective quasiequilibrium (CQE) state by day 1. The low-mid level maximum total wind, which is calculated 
using the 1.18-6.25 km vertically averaged velocity vector, gradually climbs to $\sim 80 \mathrm{~m} \mathrm{~s}^{-1}$ (Fig. 12a). By day 35, the last major merger event has finished. The later evolution is the focus of Fu and O'Neill (2021), which will not be discussed in this paper. In appendix D, we use the quasisteady rainfall rate value before day 35 to estimate the column latent heating, and use radiativeconvective equilibrium assumption to link it to the low-mid level radiative cooling rate, which yields $-\delta_{0}^{-1} \approx 11.3$ days. It indicates that day 35 corresponds to $t^{\prime} \approx 3.1$.

\section{b. The stochastic regime in the $3 D$ model}

First, we consider the establishment of the vorticity quasi-equilibrium (VQE). Using $h_{E}=800$ $\mathrm{m}, H=5 \mathrm{~km}$, (14) yields a VQE timescale of $T_{0} \approx 1.3$ day, which is comparable to the $\sim 1$ day adjustment time to CQE measured with the domain-average rainfall rate (Fig. 12c). We consider that the CQE is established once the boundary layer quasi-equilibrium (Raymond 1995) is established. This is because, the tropospheric part of the initial sounding is already in equilibrium, and the system only needs to wait until the cold pool trigger chain in the boundary layer is established. The rough overlap of $T_{0}$ with the convective adjustment time scale ( $\sim 1$ day) should be coincidental. However, we consider CQE should be established before VQE, because convection is the source of vorticity. Figure 13 shows that the power spectrum of $\omega$ amplifies in a roughly similar shape within day 1 . The spectrum shape is a mixed signal of the monopoles produced by stretching and the dipoles produced by the tilting of horizontal vorticity. The latter is omitted in our barotropic model and theory. This "shielding" effect reduces the amplitude of the low wavenumber part. Despite the deviation, the cloud-permitting simulation does possess a stochastic regime within $\sim$ day 1, which provides a initial condition for the following deterministic growth. 
Next, we consider the deterministic growth between day 1 and day 35 . The standard deviation of the spatially-filtered low-mid level vertical vorticity $(1.18-6.25 \mathrm{~km}$ vertically averaged, $\operatorname{std}(\bar{\omega}))$ shows signals of growth much earlier than day 35 (Fig. 12b). The standard deviation of the 100$\mathrm{km}$ filtered $\omega$ shows a $\sim 8.7$ day growth timescale before day $35\left(t^{\prime} \lesssim 4\right)$. Three sub-regimes are identified, as are discussed below.

The vorticity patches grow stationarily with little change of flow pattern between day 1 and day 15 (Fig. 14). It shows that the deterministic physical feedback works at a length scale down to as small as $30 \mathrm{~km}$. We hypothesize that this short-range organization is due to the nonlocal convective triggering by cold pools. The approximately stationary flow pattern, as well as the shared $\sim 8.7$ days growth timescale between the std $(\bar{\omega})$ with $30 \mathrm{~km}$ - and $100 \mathrm{~km}$-filter, indicate that the growth rate of $\operatorname{std}(\bar{\omega})$ can be used to measure the growth rate of $\langle\omega\rangle$. This corresponds to the length scale-invariant entrainment timescale $\tau_{e}$. Using (33), we get $\eta \approx 1.17$. This is not far from the estimation of $\eta$ using the vertical structure of equivalent potential temperature (Schecter and Dunkerton 2009).

There is vigorous vortex merger after day 15 . The vorticity amplitude is large enough to drive eddying motion. This transition is shown in $\operatorname{std}(\omega)$ (Fig. 12b) where the $\operatorname{std}(\bar{\omega})$ with $30 \mathrm{~km}$-filter transits from a growing to a platform stage by day 15 , and then transits to a second growing stage by day 25 . Meanwhile, the $\operatorname{std}(\bar{\omega})$ with $100 \mathrm{~km}$-filter grows steadily. Fig. 15 shows that day 15 - day 25 is an upscale-growing stage with vigorous merger but slow amplitude growth. We have not figured out the reason why the $30 \mathrm{~km}$-filtered $\omega$ does not significantly grow in amplitude at the platform stage. 
Between day 25 and day 35, the $30 \mathrm{~km}$ scale clusters merge into at least 100-km scale superclusters and grow in magnitude (Fig. 16). The $100 \mathrm{~km}$-and-above scale explains most of the $\operatorname{std}(\bar{\omega})$ (Fig. 12b). At $l \gtrsim 100 \mathrm{~km}$ scale, the vorticity evolves synchronously with the surface cold core which represents convective activity. The vorticity also evolves synchronously with the column precipitable water (PW), because convection is the source of both quantities. Thus, the positive feedbacks related to water vapor (environmental moistening and radiative cooling) should contribute to the vorticity-convection relationship. This is implicitly embedded in $\eta$. Because (C2) shows $\mathrm{Kn} \propto \bar{\omega}$ and (C3) shows $\min \{\mathrm{Kn}\} \propto l$, whether the quasi-random convection-vorticity relationship exists for smaller $l$ or earlier time (e.g. $t<20$ day for $l=30 \mathrm{~km}$ ) is hard to answer due to the insufficient sampling.

There is an interesting episode between day 30 and day 35 . By day 30 , the convective patches have organized into a dominant cyclonic vortex (Fig. 16d). However, the anti-cyclonic vortex of the non-convective region elongates the cyclone into a few pieces, which merge again by day 36 . We have not observed any similar episode in the barotropic simulation.

\section{d. Comparison with the theoretical model}

Now that our mixed stochastic-deterministic argument qualitatively agrees with the cloudpermitting simulation, we further make some quantitative comparison. A numerical integration of the ODE system (31) and (32), with the $\omega^{-}$in (32) being calculated with (38), is performed with $r_{u}=2 \mathrm{~km}, l=100 \mathrm{~km}, h_{E}=800 \mathrm{~m}, H=5 \mathrm{~km}, \eta=1.2, f_{0}=10^{-4} \mathrm{~s}^{-1},-\delta_{0}^{-1}=11.3$ day, $\lambda=2$ and $\alpha_{m}=1.6$, as is shown in Fig. 17. Here we use $V_{u p}=2 \mathrm{~m} \mathrm{~s}^{-1}$ in calculating $V_{m}$, based on the equilibrium surface wind in Fig. 12a. The predicted low-mid level maximum total wind roughly agrees with the cloud-permitting simulation. The calculation also shows that the vortex merger significantly influences $\langle\omega\rangle$ after $t^{\prime}=2$. 
The lesson to learn from the cloud-permitting simulation is that, there is no single dominant length scale for the convection-vorticity relationship. This challenges the single-length-scale filter scheme used in our barotropic model.

\section{Discussion}

We present a barotropic model with a mixed stochastic-deterministic convective parameterization to understand the formation of a tropical depression, which is the precursor of a tropical cyclone. The fully nonlinear simulation of this model can reproduce the genesis of a strong tropical depression-like vortex from initially random convection. At first, vorticity is produced via stretching by the randomly seeded convection. The repetitive stretching on lucky areas produces some high vorticity patches. As the vorticity anomaly becomes large enough, it is acted on by a Gaussian filter which preferentially seeds convection in high vorticity regions. This produces vortex clusters which self-amplify first and then merge with each other to form a single strong vortex. The filter implicitly represents the nonlocal convective trigger by cold pool and gravity wave. This positive feedback on the filtered vorticity conceptually represents the Ekman pumping (positive vorticity region induces boundary layer convergence), the cloud-radiation feedback and WISHE.

The filter not only sets a system length scale by expanding the convective region against the shrinking effect of convergence, but also spans a spatio-temporal cube in which the probability of convection genesis is smooth. This renders a relatively deterministic mesoscale, where the vortex clusters can be modelled with a dynamical system that depicts the random collision-coalescence process. The initial condition of the dynamical system is provided by the noisy earlier phase, which is denoted as "the stochastic regime". If the vorticity quasi-equilibrium state is reached 
before the positive feedback becomes significant, we can estimate the initial condition by using the shape-invariant property of the Fourier spectrum under random superposition.

The average vorticity within the strongest cluster $\langle\omega\rangle$, which is closely related to the maximum wind, is predicted to be larger for a smaller filter length scale $l$, larger feedback parameter $\eta$, larger convective intermittency (accumulated convergence in an updraft) $-\Delta h / H$, and larger Coriolis parameter $f_{0}$ (if Ekman layer height $h_{E}$ and $\eta$ are independent of $f_{0}$ ).

The theory is compared to a cloud-permitting simulation of tropical cyclogenesis over uniform sea surface temperature. Using Gaussian spatial filters with different length scales, we have identified a stochastic regime (day 0 to day 1 ), a deterministic regime with a stationary growing subregime (day 1 to day 15), a second sub-regime with vigorous vortex merger (day 15 to day 25), and a third sub-regime that marks the formation-by-merger and amplification of a larger-than-100 $\mathrm{km}$ super cluster (day 25 to day 35 ). The evolution of the low-mid level maximum total wind is qualitatively captured by the theoretical model.

However, the seemingly non-unique candidate of $l$ shows that a single nonlocal convective triggering length scale is insufficient. In a follow-up paper, we will investigate the physical basis of using filter to represent the nonlocal trigger by establishing a theory of the convective trigger chain.

Even if no further complexity is added, the present theoretical model can be improved in the following aspects:

- An extension to consider the size and circulation spectrum of the vortex clusters is desired. Especially, a heterogeneous treatment of the vortex clusters in the stochastic regime is the key to resolve the underestimated sensitivity of $\omega_{0}$ to $l$. 
- A collision-coalescence model of vortex-sink system (Novikov and Novikov 1996) rather than a pure vortex system is needed to account for the mesoscale convergent flow, which is especially important for a low latitude problem. This might help establish a unified understanding of spontaneous tropical cyclogenesis and convective self-aggregation (Muller and Romps 2018; Carstens and Wing 2020).

The barotropic model can also be improved. The laminar Ekman layer formulation in this paper, which directly links vorticity to Ekman pumping, provides a clean treatment of the physical feedback. However, to make the model more useful, it should be updated to a turbulent Ekman layer scheme which uses bulk aerodynamic formulae as the boundary condition. An extension to two vertical levels can be used to study the interaction between the stratiform and convective cloud, which is the key to understand the role of mid-level vortex in spinning up the low-level circulation (Bell and Montgomery 2019).

\section{APPENDIX A}

\section{From the filter length scale $l$ to the system length scale $l_{*}$}

Here we explore how the nonlocal convective trigger (represented by a Gaussian filter) cooperates with convergence to set the system length scale $l_{*}$.

The vorticity equation (1) is a stochastic nonlinear integro-differential equation. Imposing a filter on (1), we get the mesoscale vorticity equation:

$$
\frac{\partial \bar{\omega}}{\partial t}+\overline{\mathbf{u} \cdot \nabla \omega}=-\overline{\left(\omega+f_{0}\right) \delta}
$$


Substituting in (12), we neglect the sub-filter terms in (A1) to get:

$$
\begin{aligned}
\frac{\partial \bar{\omega}}{\partial t}+\overline{\mathbf{u}} \cdot \nabla \bar{\omega} & \approx \underbrace{\overline{\bar{\omega}}\left(\bar{\omega}+f_{0}\right) \eta \mathrm{E}}_{\text {updraft }}-\underbrace{\bar{\omega}\left(\bar{\omega}+f_{0}\right) \mathrm{E}}_{\text {spin down }} \\
& \approx \overline{\bar{\omega}} \underbrace{\overline{\left(\bar{\omega}+f_{0}\right)(\eta-1)} \mathrm{E} .}_{\tau^{-1}}
\end{aligned}
$$

To facilitate the math, we have added a filter on the spin down term to make it work at the same scale as the updraft. This approximation weakens the damping effect on scale contraction, and therefore causes an underestimation of the system scale. We have also introduced a growth time scale $\tau^{-1} \equiv\left(\bar{\omega}+f_{0}\right)(\eta-1)$ E to denote the vorticity magnitude growth due to stretching. Supposing $\tau$ is a constant, (A2) is simplified with a Taylor expansion on the Gaussian filter term:

$$
\frac{\partial \bar{\omega}}{\partial t}+\overline{\mathbf{u}} \cdot \nabla \bar{\omega} \approx \frac{\overline{\bar{\omega}}}{\tau} \approx \frac{\bar{\omega}}{\tau}+D \nabla^{2} \bar{\omega} \text { with } \quad D=\frac{l^{2}}{4 \tau} .
$$

If the advection term is further neglected, (A3) reduces to a form identical to the column water vapor equation used by Windmiller and Craig (2019), who used the filter to represent the expansion of cloud size during a convective lifetime. In our model, the diffusivity $D$ represents the encroachment of non-convective region by cold pools and gravity waves generated at the rim of a convective cluster.

When $\bar{\omega} \ll f_{0}$, there is $\tau^{-1} \gg-\delta$, with $\delta$ obeying (12). The advection can be neglected, making the system linear. The basic length scale $l_{*}$ should be

$$
l_{*}=\left\{\begin{array}{l}
(4 D t)^{1 / 2},\left.\quad l_{*}\right|_{t=T_{0}}<(4 D \tau)^{1 / 2}, \\
(4 D \tau)^{1 / 2} \sim l, \quad \text { else. }
\end{array}\right.
$$

The first scaling has been used by Windmiller and Craig (2019) in their coarsening model where the initial condition is a highly fine-grained noise. It agrees with their non-rotating cloudpermitting simulation at the early stage. The second scaling, which denotes scale saturation, is the neutral wavelength of the normal mode solution of (A3). Our theory only predicts the mesoscale, 
so the initial length scale of $\bar{\omega}$ for the deterministic regime is $l$. Thus, the system length scale will stay at $l$ according to the second scaling of (A4). Though this conclusion is simple, there is a more complicated process behind. The merger as a nonlinear factor can increase the size of the cluster's central vortex, which is smaller than $l$ at the beginning of the deterministic regime. The convergent flow in the later evolution will constrain the size of a cluster, as well as its embedded central vortex, to be no larger than $l$. This is discussed below.

At the later stage where $\bar{\omega} \gtrsim f_{0}$, there is $\tau^{-1} \sim-\delta$. The convergent flow makes the positive vorticity aggregate into compact convective clusters and a widespread negative vorticity region where convection seldom occurs. Windmiller and Craig (2019) also briefly mentioned the transition from a diffusion-dominated to an advection-dominated regime of moisture in non-rotating convective self-aggregation, but have not presented any scaling on the length scale. Our derivation begins by noticing that the normal mode argument no longer works, with the convective and non-convective region having different length scales. We focus on the length scale of the convective region, and seek for a Lagrangian treatment. The $\bar{\omega} / \tau$ and $D \nabla^{2} \bar{\omega}$ terms show that the convective area expands linearly with time at a rate of $l^{2} / \tau$. The advection term makes the area shrink at a timescale of $-\delta^{-1}$ at the same time. Let the $l_{*}^{2}$ tendency caused by the filter be $\left(d l_{*}^{2} / d t\right)_{l}=l^{2} / \tau \sim-l^{2} \delta$ and that by convergence as $\left(d l_{*}^{2} / d t\right)_{\delta}=l_{*}^{2} \delta$. The sum of the tendency leads to an ODE of $l_{*}^{2}$ :

$$
\frac{d l_{*}^{2}}{d t} \sim\left(\frac{d l_{*}^{2}}{d t}\right)_{l}+\left(\frac{d l_{*}^{2}}{d t}\right)_{\delta}=\delta\left(l_{*}^{2}-l^{2}\right)
$$

It yields a stable equilibrium radius $l$ with a relaxation time scale of $-\delta^{-1}$. Thus, $l$ serves as the system length scale at all stages.

The influence of $-\Delta h / H$ on the system length scale is briefly discussed. Fu and O'Neill (2021) showed that a higher $-\Delta h / H$ renders a more coarse-grained updraft seeding mode, where the more persistent vorticity stretching leads to more high vorticity air columns. These eddies efficiently 
aggregate the surrounding vorticity patches and make the vortex cluster more compact. Their effects are stored in the sub-filtered terms, which have been neglected in (A2). Figure 6 shows that the system length scale of the $-\Delta h / H=0.4$ case is about twice the $-\Delta h / H=1.6$ (reference) case. The quantitative modelling of the eddy's effect on the length scale is left for future work.

\section{APPENDIX B}

\section{The signal-to-noise ratio of the convective scheme}

The signal-to-noise ratio (SNR) of the seeding scheme (9) is defined as the ratio of updraft-induced divergence anomaly to its basic state value. The SNR at the vorticity equilibrium time $T_{0}$ is derived with (9), (11) and (25):

$$
\begin{aligned}
\left.\mathrm{SNR}\right|_{t=T_{0}} & \equiv \frac{\left.\delta_{u}\right|_{\omega=\omega_{0}}-\delta_{0}}{\delta_{0}} \\
& =-\eta \mathrm{E} \frac{\omega_{0}}{\delta_{0}} \\
& =-\lambda \eta \mathrm{E} \frac{f_{0}}{\delta_{0}}\left(\delta_{0} T_{0} \frac{\Delta h}{H} \frac{r_{u}^{2}}{\pi l^{2}}\right)^{1 / 2} .
\end{aligned}
$$

It involves all the five nondimensional parameters. The separation between the stochastic and deterministic regime requires $\left.\mathrm{SNR}\right|_{t=T_{0}} \ll 1$. The reference parameter yields $\left.\mathrm{SNR}\right|_{t=T_{0}} \approx 0.24$, so the separation is roughly valid.

\section{APPENDIX C}

\section{A measure of the mesoscale determinacy}

A high mesoscale determinacy means there is a sufficiently large number of updrafts falling in a spatio-temporal cube $l^{2} T$, where $T$ is the shortest time scale of $\rho_{u}$ (or $\left.\bar{\omega}\right)$ and $l$ is its smallest spatial scale. We denote the updraft number in the cube, after subtracting the part that balances 


$$
\begin{aligned}
\max \{\mathrm{Kn}\} & =\left.\mathrm{Kn}\right|_{t=T_{0}} \\
& =\lambda^{-1} \frac{\eta-1}{\eta}\left(-\delta_{0} T_{0}\right)^{-1 / 2}\left(-\frac{\Delta h}{H}\right)^{1 / 2}\left(\frac{r_{u}^{2}}{\pi l^{2}}\right)^{1 / 2},
\end{aligned}
$$

which yields $\max \{\mathrm{Kn}\} \approx 0.26$ for the reference test $(l=60 \mathrm{~km})$. Thus, the mesoscale is roughly deterministic. Otherwise, some stochastic perturbation on the vortex cluster motion and strength needs to be considered (e.g. Chavanis 2008).

The determinacy requires $\mathrm{Kn} \ll 1$. The original definition of Knudsen number is the ratio of the mean free path of gas molecules to the length scale of interest, which measures the degree to which the continuum assumption is valid (Batchelor 2000). Let $T=\tau_{e}$ be a rough estimate of the characteristic timescale. Substituting (9), (10) and (33) into (C1), we get:

$$
\mathrm{Kn}=-\frac{\Delta h}{H} \frac{\pi r_{u}^{2}}{l^{2}} \frac{\eta-1}{\eta} \frac{f_{0}}{\bar{\omega}} \leq-\frac{\Delta h}{H} \frac{\pi r_{u}^{2}}{l^{2}} \frac{f_{0}}{\bar{\omega}} .
$$

Note that Kn does not depend on E. The determinacy is predicted to be higher for a larger $\bar{\omega} / f_{0}$, which makes the feedback signal stronger, and a lower $r_{u}$ and $-\Delta h / H$ which raise the convective number density. It might be somewhat surprising that a smaller $\eta$ reduces Kn. This is because, as $\eta$ approaches unity from above, the growth time scale $\tau_{e} \rightarrow \infty$, but the seeding rate is still finite and balances with Ekman spin down.

Next, we estimate the value of Kn of our reference test simulation. Because $\bar{\omega}$ increases in the deterministic regime, the maximum Kn occurs at the beginning $\left(t=T_{0}\right)$ is calculated by substituting (25) into (C2): 
Before day 35, the domain-averaged rainfall rate $R$ has a quasi-steady value of $\approx 0.32 \mathrm{~cm} \mathrm{day}^{-1}$ $\left(4.63 \times 10^{8} \mathrm{~m} \mathrm{~s}^{-1}\right)$. Suppose all the condensation heating is exerted on the low-mid level, the low-mid layer net condensation heating rate (unit: $\mathrm{K} \mathrm{s}^{-1}$ ) is $Q_{c}=R\left(\rho_{l} / \rho_{a}\right)\left(L_{v} / c_{p}\right) H^{-1}$, where $\rho_{l}=10^{3} \mathrm{~kg} \mathrm{~m}^{-3}$ is liquid water density, $\rho_{a}=0.9 \mathrm{~kg} \mathrm{~m}^{-3}$ is the low-mid level air density, $L_{v}=$ $2.5 \times 10^{6} \mathrm{~J} \mathrm{~kg}^{-1}$ is evaporation latent heat, $c_{p}=1004 \mathrm{~J} \mathrm{~K}^{-1} \mathrm{~kg}^{-1}$. Considering that the net latent heating is balanced by radiative cooling, and using $\Delta \theta=20 \mathrm{~K}$, we have:

$$
\delta_{0}=-\delta_{\text {rad }}=-\frac{Q_{c}}{\Delta \theta}=-R \frac{L_{v}}{c_{p} \Delta \theta} \frac{\rho_{l}}{\rho_{a}} \frac{1}{H},
$$

which yields $-\delta_{0}^{-1} \approx 11.3$ days $\left(1 \times 10^{-6} \mathrm{~s}^{-1}\right)$ and $Q_{c} \approx 1.77 \mathrm{~K} \mathrm{day}^{-1}$.

Acknowledgments. The MATLAB code for the numerical simulation, postprocessing, a math note, and movies of the barotropic and cloud-permitting simulation can be downloaded here: https://stanford.box.com/s/unuwb6dswuoz49hl9vxm2qrq8ffifsgo. We are grateful to Prof. Da Yang at UC Davis, Prof. Zhaohua Wu at Florida State University, and Dr. Yan Liu at Nanjing University for helpful discussion. We thank Stanford University and Stanford Research Computing Center for providing funding and computational resources.

\section{References}

Ahmed, F., and J. D. Neelin, 2019: Explaining scales and statistics of tropical precipitation clusters with a stochastic model. J. Atmos. Sci., 76 (10), 3063-3087.

AMS-Glossary, 2012: Glossary of meteorology, American Meteorological Society. URl: http://glossary.ametsoc.org.

Batchelor, G., 2000: An introduction to fluid dynamics. Cambridge university press. 
Bell, M. M., and M. T. Montgomery, 2019: Mesoscale processes during the genesis of Hurricane Karl (2010). J. Atmos. Sci., 76 (8), 2235-2255.

Brenowitz, N., Y. Frenkel, and A. Majda, 2016: Non-local convergence coupling in a simple stochastic convection model. Dyn. Atmos. Oceans, 74, 30-49.

Bretherton, C. S., P. N. Blossey, and M. Khairoutdinov, 2005: An energy-balance analysis of deep convective self-aggregation above uniform SST. J. Atmos. Sci., 62 (12), 4273-4292.

Bryan, G. H., and J. M. Fritsch, 2002: A benchmark simulation for moist nonhydrostatic numerical models. Mon. Wea. Rev., 130 (12), 2917-2928.

Carnevale, G., J. McWilliams, Y. Pomeau, J. Weiss, and W. Young, 1991: Evolution of vortex statistics in two-dimensional turbulence. Phys. Rev. Lett., 66 (21), 2735.

Carstens, J. D., and A. A. Wing, 2020: Tropical cyclogenesis from self-aggregated convection in numerical simulations of rotating radiative-convective equilibrium. J. Adv. Model. Earth Syst., 12 (5), e2019MS002 020.

Charney, J. G., and A. Eliassen, 1964: On the growth of the hurricane depression. J. Atmos. Sci., $21(1), 68-75$.

Chavanis, P.-H., 2008: Two-dimensional brownian vortices. Physica A, 387 (28), 6917-6942.

Craig, G., and J. Mack, 2013: A coarsening model for self-organization of tropical convection. $J$. Geophys. Res., 118 (16), 8761-8769.

Cross, M. C., and P. C. Hohenberg, 1993: Pattern formation outside of equilibrium. Rev. Mod. Phys., 65 (3), 851. 
Davis, C. A., 2015: The formation of moist vortices and tropical cyclones in idealized simulations. J. Atmos. Sci., 72 (9), 3499-3516.

Dunkerton, T., M. Montgomery, and Z. Wang, 2009: Tropical cyclogenesis in a tropical wave critical layer: easterly waves. Atmos. Chem. Phys., 9 (15).

Emanuel, K. A., 1986: An air-sea interaction theory for tropical cyclones. Part I: Steady-state maintenance. J. Atmos. Sci., 43 (6), 585-605.

Enagonio, J., and M. T. Montgomery, 2001: Tropical cyclogenesis via convectively forced vortex rossby waves in a shallow water primitive equation model. J. Atmos. Sci., 58 (7), 685-706.

Fang, J., and F. Zhang, 2011: Evolution of multiscale vortices in the development of Hurricane Dolly (2008). J. Atmos. Sci., 68 (1), 103-122.

Favier, B., L. Silvers, and M. Proctor, 2014: Inverse cascade and symmetry breaking in rapidly rotating Boussinesq convection. Phys. Fluids, 26 (9), 096605.

Fu, H., and M. O'Neill, 2021: The role of random vorticity stretching in tropical depression genesis. EarthArXiv: https://eartharxiv.org/repository/view/2191/.

Guervilly, C., D. W. Hughes, and C. A. Jones, 2014: Large-scale vortices in rapidly rotating Rayleigh-Bénard convection. J. Fluid Mech., 758, 407-435.

Haerter, J. O., 2019: Convective self-aggregation as a cold pool-driven critical phenomenon. Geophys. Res. Lett., 46 (7), 4017-4028.

Haerter, J. O., S. J. Böing, O. Henneberg, and S. B. Nissen, 2019: Circling in on convective organization. Geophys. Res. Lett., 46 (12), 7024-7034. 
Hartmann, D. L., J. R. Holton, and Q. Fu, 2001: The heat balance of the tropical tropopause, cirrus, and stratospheric dehydration. Geophys. Res. Lett., 28 (10), 1969-1972.

Hendricks, E. A., M. T. Montgomery, and C. A. Davis, 2004: The role of "vortical" hot towers in the formation of Tropical Cyclone Diana (1984). J. Atmos. Sci., 61 (11), 1209-1232.

Hottovy, S., and S. N. Stechmann, 2015: A spatiotemporal stochastic model for tropical precipitation and water vapor dynamics. J. Atmos. Sci., 72 (12), 4721-4738.

Houze Jr, R. A., W.-C. Lee, and M. M. Bell, 2009: Convective contribution to the genesis of Hurricane Ophelia (2005). Mon. Wea. Rev., 137 (9), 2778-2800.

Jeevanjee, N., and D. M. Romps, 2013: Convective self-aggregation, cold pools, and domain size. Geophys. Res. Lett., 40 (5), 994-998.

Khairoutdinov, M., and K. Emanuel, 2013: Rotating radiative-convective equilibrium simulated by a cloud-resolving model. J. Adv. Model. Earth Syst., 5 (4), 816-825.

Kilroy, G., R. K. Smith, and M. T. Montgomery, 2017: A unified view of tropical cyclogenesis and intensification. Quart. J. Roy. Meteor. Soc., 143 (702), 450-462.

Langhans, W., and D. M. Romps, 2015: The origin of water vapor rings in tropical oceanic cold pools. Geophys. Res. Lett., 42 (18), 7825-7834.

Liu, C., and M. W. Moncrieff, 2004: Effects of convectively generated gravity waves and rotation on the organization of convection. J. Atmos. Sci., 61 (17), 2218-2227.

Liu, Y., Z.-M. Tan, and Z. Wu, 2019: Noninstantaneous wave-CISK for the interaction between convective heating and low-level moisture convergence in the tropics. J. Atmos. Sci., 76 (7), 2083-2101. 
Mapes, B. E., 1993: Gregarious tropical convection. J. Atmos. Sci., 50 (13), 2026-2037.

McWilliams, J. C., 1990: The vortices of two-dimensional turbulence. J. Fluid Mech., 219, 361385.

Mihos, J. C., and L. Hernquist, 1996: Gasdynamics and starbursts in major mergers. Astrophys. $J$, 464, 641.

Montgomery, M., M. Nicholls, T. Cram, and A. Saunders, 2006: A vortical hot tower route to tropical cyclogenesis. J. Atmos. Sci., 63 (1), 355-386.

Montgomery, M. T., and R. K. Smith, 2014: Paradigms for tropical cyclone intensification. Australian Meteorological and Oceanographic Journal, 64 (1), 37-66.

Muller, C. J., and D. M. Romps, 2018: Acceleration of tropical cyclogenesis by self-aggregation feedbacks. Proc. Natl. Acad. Sci. (USA), 115 (12), 2930-2935.

Neelin, J. D., and I. M. Held, 1987: Modeling tropical convergence based on the moist static energy budget. Mon. Wea. Rev., 115 (1), 3-12.

Novikov, A., and E. Novikov, 1996: Vortex-sink dynamics. Phys. Rev. E, 54 (4), 3681.

Ooyama, K., 1969: Numerical simulation of the life cycle of tropical cyclones. J. Atmos. Sci., $26(1), 3-40$.

O’Neill, M. E., K. A. Emanuel, and G. R. Flierl, 2016: Weak jets and strong cyclones: Shallowwater modeling of giant planet polar caps. J. Atmos. Sci., 73 (4), 1841-1855.

Raymond, D. J., 1995: Regulation of moist convection over the west pacific warm pool. J. Atmos. Sci., 52 (22), 3945-3959. 
Raymond, D. J., S. L. Sessions, and Ž. Fuchs, 2007: A theory for the spinup of tropical depressions. Quart. J. Roy. Meteor. Soc., 133 (628), 1743-1754.

Riley, J. J., and M.-P. Lelong, 2000: Fluid motions in the presence of strong stable stratification. Annu. Rev. Fluid Mech., 32 (1), 613-657.

Ritchie, E. A., and G. J. Holland, 1997: Scale interactions during the formation of Typhoon Irving. Mon. Wea. Rev., 125 (7), 1377-1396.

Ruppert, J. H., A. A. Wing, X. Tang, and E. L. Duran, 2020: The critical role of cloud-infrared radiation feedback in tropical cyclone development. Proc. Natl. Acad. Sci. (USA), 117 (45), $27884-27892$.

Sansón, L. Z., and G. Van Heijst, 2000: Nonlinear ekman effects in rotating barotropic flows. $J$. Fluid Mech., 412, 75-91.

Schecter, D. A., 2011: Evaluation of a reduced model for investigating hurricane formation from turbulence. Quart. J. Roy. Meteor. Soc., 137 (654), 155-178.

Schecter, D. A., 2016: Development and nondevelopment of binary mesoscale vortices into tropical cyclones in idealized numerical experiments. J. Atmos. Sci., 73 (3), 1223-1254.

Schecter, D. A., 2017: A computational study on the nature of meso- $\beta$ scale vortex coalescence in a tropical atmosphere. J. Adv. Model. Earth Syst., 9 (2), 1366-1398.

Schecter, D. A., and T. J. Dunkerton, 2009: Hurricane formation in diabatic ekman turbulence. Quart. J. Roy. Meteor. Soc., 135 (641), 823-838.

Showman, A. P., 2007: Numerical simulations of forced shallow-water turbulence: Effects of moist convection on the large-scale circulation of Jupiter and Saturn. J. Atmos. Sci., 64 (9), $3132-3157$. 
Sobel, A. H., J. Nilsson, and L. M. Polvani, 2001: The weak temperature gradient approximation and balanced tropical moisture waves. J. Atmos. Sci., 58 (23), 3650-3665.

Stellmach, S., M. Lischper, K. Julien, G. Vasil, J. S. Cheng, A. Ribeiro, E. M. King, and J. M. Aurnou, 2014: Approaching the asymptotic regime of rapidly rotating convection: boundary layers versus interior dynamics. Phys. Rev. Lett., 113 (25), 254501.

Tompkins, A. M., 2001: Organization of tropical convection in low vertical wind shears: The role of cold pools. J. Atmos. Sci., 58 (13), 1650-1672.

Torri, G., and Z. Kuang, 2019: On cold pool collisions in tropical boundary layers. Geophys. Res. Lett., 46 (1), 399-407.

Trizac, E., 1998: A coalescence model for freely decaying two-dimensional turbulence. Europhys Lett., 43 (6), 671.

Vallis, G., G. Shutts, and M. Gray, 1997: Balanced mesoscale motion and stratified turbulence forced by convection. Quart. J. Roy. Meteor. Soc., 123 (542), 1621-1652.

Vallis, G. K., 2017: Atmospheric and oceanic fluid dynamics. Cambridge University Press.

Weisstein, E. W., 2021: Random walk-2-dimensional. https://mathworld.wolfram.com/RandomWalk2-1 Dimensional.html.

Windmiller, J., and C. Hohenegger, 2019: Convection on the edge. J. Adv. Model. Earth Syst., 11 (12), 3959-3972.

Windmiller, J. M., and G. C. Craig, 2019: Universality in the spatial evolution of self-aggregation of tropical convection. J. Atmos. Sci., 76 (6), 1677-1696. 
Wing, A. A., S. J. Camargo, and A. H. Sobel, 2016: Role of radiative-convective feedbacks in spontaneous tropical cyclogenesis in idealized numerical simulations. J. Atmos. Sci., 73 (7), 2633-2642.

Yang, B., and Z.-M. Tan, 2020: Interactive radiation accelerates the intensification of the midlevel vortex for tropical cyclogenesis. J. Atmos. Sci., 77 (12), 4051-4065.

Yang, D., 2020: A shallow water model for convective self-aggregation. J. Atmos. Sci., 1-41.

Yang, D., and A. P. Ingersoll, 2013: Triggered convection, gravity waves, and the MJO: A shallowwater model. J. Atmos. Sci., 70 (8), 2476-2486.

Yau, M. K., and R. R. Rogers, 1996: A short course in cloud physics. Elsevier. 


\section{LIST OF FIGURES}

Fig. 1. The trajectory of tropical cyclogenesis in the $\mathrm{Fr}-$ Ro space of geophysical fluid. $\mathrm{The} \mathrm{Fr}=$ $U / c$ is Froude number, and the Ro $=U /\left(f_{0} l_{*}\right)$ is Rossby number, with $l_{*}$ as the system length scale. The $U$ denotes the characteristic velocity, $c$ denotes gravity wave speed, $l_{*}$ denotes the system length scale. In our view, tropical cyclogenesis is a multiscale process, which starts from the small-scale thunderstorms (stratified turbulence) with small $U$ and small $l_{*}$. As the convection organizes via cold pool and gravity wave, $l_{*}$ quickly rises to the length scale of the "statistical mesoscale" $l$. The drop of Ro makes the system enter the "dynamical mesoscale". Then, $U$ gradually rises and $l_{*}$ keeps around $l$, indicating the growth of Fr and Ro at the same time. The system then leaves the "dynamical mesoscale" and grows into a mature tropical cyclone. The positions of other regimes are summarized from Riley and Lelong (2000) and Vallis (2017).

Fig. 2. A schematic diagram of the quasi-random convective seeding scheme. Convection is preferentially seeded near the high vorticity region.

Fig. 3. The unfiltered $\omega / f_{0}$ field of the index-1-run in the ensemble of the $l=60 \mathrm{~km}$ test at (a) $t_{1}^{\prime}=-\delta_{0} t=0.55$, (b) $t_{2}^{\prime}=1.54$, (c) $t_{3}^{\prime}=2.54$, (d) $t_{4}^{\prime}=3.54$, (e) $t_{5}^{\prime}=4.54$, and (f) $t_{6}^{\prime}=5.54$. . . 52

Fig. 4. The same as Fig. 3, but has been filtered with a $l=60 \mathrm{~km}$ Gaussian filter.

Fig. 5. (a)(b)(c) are the $t_{1}^{\prime}=0.55$ snapshots of $\omega / f_{0}$ for the purely random test, $l=30 \mathrm{~km}$ test and $l=45 \mathrm{~km}$ test, using the index-1-run in the ensemble. (d)(e)(f) are the corresponding $t_{4}^{\prime}=3.54$ vorticity snapshots.

Fig. 6. The same as Fig. 5, but for the filtered field $\overline{\omega / f_{0}}$ instead. (a) and (d) are the result of the purely random test processed with a $l=30 \mathrm{~km}$ filter. (b) and (e) are the $l=30 \mathrm{~km}$ test, processed with the corresponding $l$. (c) and (f) are the $l=45 \mathrm{~km}$ test, processed with the corresponding $l$.

Fig. 7. The azimuthally averaged $\omega / f_{0}$ profile centered at the maximum $\bar{\omega}$ point in the domain, for (a) $l=30 \mathrm{~km}$, (b) $l=45 \mathrm{~km}$ and (c) $l=60 \mathrm{~km}$ test respectively. The solid lines denote the ensemble-average, and the shadow denotes the \pm 1 standard deviation range. For (a), the blue, red and yellow lines denote $t^{\prime}=t_{1}^{\prime}, t_{2}^{\prime}$, and $t_{3}^{\prime}$ respectively. For (b), the blue, red and yellow lines denote $t^{\prime}=t_{2}^{\prime}, t_{3}^{\prime}$, and $t_{4}^{\prime}$ respectively. For (c), the blue, red and yellow lines denote $t^{\prime}=t_{3}^{\prime}, t_{4}^{\prime}$, and $t_{5}^{\prime}$ respectively. The sampling slot is shifted, because the system growth rate is slower for a larger $l$.

Fig. 8. (a) The total number of the maximum points in the $\bar{\omega}$ field $N=N_{v} L^{2}$. The $N_{v}$ is the spatial number density of the maximum points, which is interpreted as the number density of vortex cluster. The blue line denotes the $l=30 \mathrm{~km}$ filter test, the red line denotes the $l=45 \mathrm{~km}$ test, the yellow line denotes the $l=60 \mathrm{~km}$ test, and the purple line denotes the purely random test. The shadow denotes the \pm 1 standard deviation range of a five-member ensemble. (b) is the same as (a), but for the standard deviation of relative vorticity $\operatorname{std}(\omega)$ normalized with $f_{0}$. The time series of a test is truncated once a member in the ensemble blows up.

Fig. 9. (a) The one-dimensional power spectrum $|\hat{\omega}|$ of the index-1-run of the purely random test. It is obtained by "azimuthally" averaging $|\hat{\omega}|$ over each wavenumber circle (constant $\left.|\mathbf{k}|=\sqrt{k_{x}^{2}+k_{y}^{2}}\right)$. The solid blue line denotes $t^{\prime}=0.12\left(t^{\prime} \approx-\delta_{0} T_{0} / 4\right)$, the solid red line denotes $t^{\prime}=0.24\left(t^{\prime} \approx-\delta_{0} T_{0} / 2\right)$, and the solid red line denotes $t^{\prime}=0.47\left(t^{\prime} \approx-\delta_{0} T_{0}\right)$. The dashed purple line denotes the equilibrium spectrum predicted by (22). The diagnosed spec- 
trum at the high wavenumber end is gentler than the theoretical estimate, probably due the sharpening effect of the convergent transport. (b) The dependence of domain total vortex number $N_{v 0} L^{2}$ on filter length $l$, at $t^{\prime}=0.47\left(t^{\prime} \approx-\delta_{0} T_{0}\right)$. All the simulations are run with $l=60 \mathrm{~km}$, and the value at different $l$ is due to the change of $l$ in filtering the same set of $\omega$ data. The solid blue, red and yellow lines denote the data using $-\Delta h / H=0.4,0.8$ and 1.6 respectively. Note that $\tau_{u}$ is changed proportionally to keep the updraft-induced convergence $-\Delta h /\left(\tau_{u} H\right)$ unchanged. The yellow dashed line denotes the theoretical prediction $N_{l}=L^{2} / \pi l^{2}$. (c) is the same as (b), but for benchmarking the theoretical $\omega_{0} / f_{0}$ at $-\Delta h / H=0.4,0.8$ and 1.6 (dashed blue, red and yellow lines). The simulation counterpart (solid lines) uses $\langle\omega\rangle / f_{0}$, where $\langle\omega\rangle$ is defined as the area average within $2 l$ radius of the maximum $\bar{\omega}$ point (the strongest vortex cluster). The motivation of this choice is explained in section $3 \mathrm{c}$.

Fig. 10. The comparison between the barotropic simulation (denoted as the solid lines) and the theory (the numerical solution of the ODE system, denoted as the dashed lines) on the time series of $\langle\omega\rangle / f_{0}$ (the left column), $\omega^{-} / f_{0}$ (the middle column) and the maximum wind $V_{m}$ (the right column). The first row (a)(b)(c) is for different $l$, with the blue, red, and yellow lines denoting the five-run ensemble average of $l=30 \mathrm{~km}, l=45 \mathrm{~km}, l=60 \mathrm{~km}$ and purely random test (equivalent to $l \rightarrow \infty$ ) respectively. The shadow denotes the corresponding \pm 1 standard deviation range of the barotropic simulation. The second row (d)(e)(f) is for $\eta=1.2$ (the blue line), $\eta=1.6$ (the red line, also the reference test), and $\eta=2$ test (the yellow line). The third row $(\mathrm{g})(\mathrm{h})(\mathrm{i})$ is for $-\Delta h / H=0.4$ (the blue line), 0.8 (the red line), and 1.6 test (the yellow line, also the reference test). The forth row $(\mathrm{j})(\mathrm{k})(\mathrm{l})$ is for the half Coriolis parameter $f_{0}=0.5 \times 10^{-4}$ test (the blue line), the reference test (the red line), and the 1.5 times Coriolis parameter $f_{0}=1.5 \times 10^{-4} \mathrm{~s}^{-1}$ test (the yellow line). The time series of a test is truncated once a member in the ensemble blows up.

Fig. 11. (a) The evolution of $\langle\omega\rangle / f_{0}$ predicted by the theory for $l=30,45$ and $60 \mathrm{~km}$ tests, which are denoted as the blue, red and yellow lines respectively. The solid lines denote the numerical solution of the ODE system, and the dashed lines denote the approximate analytical solution. (b) is the same as (a), but for the total vortex number in the domain $N_{v} L^{2}$. (c) is the same as (a), but for the nondimensional merger timescale $-\delta_{0} \tau_{m}$.

Fig. 12. (a) The blue line denotes the low-mid level maximum total wind calculated using the 1.18$6.25 \mathrm{~km}$ vertically averaged velocity vector, and the red line denotes the domain-average rainfall rate (unit: $\mathrm{cm} \mathrm{day}^{-1}$ ). (b) The standard deviation of low-mid level vertical vorticity $\omega$ (averaged between 1.18-6.25 km height). The blue line denotes the domain-average time series. The red, yellow and purple lines denote the standard deviation of the vorticity filtered with $l=10 \mathrm{~km}, 30 \mathrm{~km}$ and $100 \mathrm{~km}$. (c) and (d) are the zoom-in plots of (a) and (b) for the first two days.

Fig. 13. (a) The power spectrum of $\omega / f_{0}$ at $t=0.25$ days (the blue line), $t=0.50$ days (the red line), and $t=1.00$ days (the yellow line). It is calculated in the same way as Fig. 9a. The dashed purple line is a theoretical estimation of the equilibrium spectrum using (22), with the parameters listed in section $6 \mathrm{~d}$ (e.g. $\left.r_{u}=2 \mathrm{~km}\right)$. (b) A zoom-in plot of the central region $\omega / f_{0}$ at $t=1.00$ days.

Fig. 14. A zoom-in plot of the $\bar{\omega} / f_{0}$ which uses a $30 \mathrm{~km}$ Gaussian filter, at (a) $t=7$ days, (b) $t=9$ days, and (c) $t=11$ days. They show the stationary growth sub-regime in the deterministic regime.

Fig. 15. A plot of the $\bar{\omega} / f_{0}$ which uses a $30 \mathrm{~km}$ Gaussian filter, at (a) $t=15$ days, (b) $t=20$ days, and (c) $t=25$ days. They show the merger sub-regime in the deterministic regime. 
Fig. 16. The $100 \mathrm{~km}-\mathrm{Gaussian}$ filtered quantities. The first column denotes the nondimensional filtered low-mid level vertical vorticity $\left(\bar{\omega} / f_{0}\right)$. The second column denotes the opposite of the filtered disturbance potential temperature $-\overline{\theta^{\prime}}$ at $z=25 \mathrm{~m}$ (having subtracted the domainaverag value). The third column denotes the filtered column precipitable water (PW, unit: $\mathrm{m})$. The first, second, third and fourth row denotes the data at $t=25$ days, 30 days, 35 days and 40 days respectively. The range of the colorbar is not controlled, because we focus on the pattern.

Fig. 17. The numerical solution of the ODE system of the mixed stochastic-deterministic theory. (a) The characteristic vorticity of the vortex cluster $\langle\omega\rangle / f_{0}$, (b) the environmental vorticity $\omega^{-} / f_{0}$, and (c) the maximum wind $V_{m}$. The red line denotes the numerical solution that arbitrarily turns off the merger term (by setting $\alpha_{m} \rightarrow \infty$ ), and the blue line denotes the normal solution with the merger term. Note that the $\omega^{-} / f_{0}$ of the two simulations is identical.

Fig. A1. The azimuthal average $\omega / f_{0}$ profile centered at the maximum $\bar{\omega}$ point at $t_{5}^{\prime}=4.54$. The blue line denotes the five-member ensemble average of the $-\Delta h / H=0.4$ test, the red line denotes the $-\Delta h / H=0.8$ test, and the yellow line denotes the $-\Delta h / H=1.6$ test (reference test). The shadow of corresponding color denotes the \pm 1 standard deviation. 


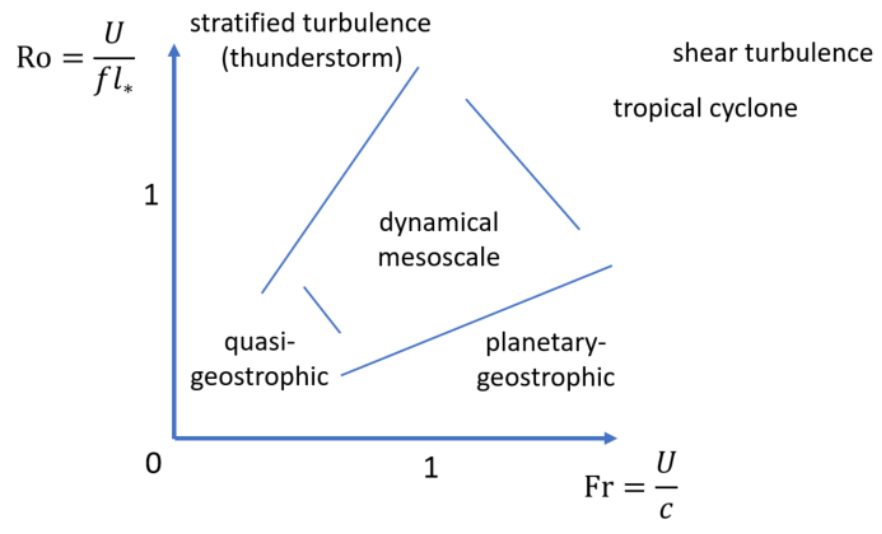

FIG. 1. The trajectory of tropical cyclogenesis in the $\mathrm{Fr}-$ Ro space of geophysical fluid. The $\mathrm{Fr}=U / c$ is Froude number, and the Ro $=U /\left(f_{0} l_{*}\right)$ is Rossby number, with $l_{*}$ as the system length scale. The $U$ denotes the characteristic velocity, $c$ denotes gravity wave speed, $l_{*}$ denotes the system length scale. In our view, tropical cyclogenesis is a multiscale process, which starts from the small-scale thunderstorms (stratified turbulence) with small $U$ and small $l_{*}$. As the convection organizes via cold pool and gravity wave, $l_{*}$ quickly rises to the length scale of the "statistical mesoscale" $l$. The drop of Ro makes the system enter the "dynamical mesoscale". Then, $U$ gradually rises and $l_{*}$ keeps around $l$, indicating the growth of Fr and Ro at the same time. The system then leaves the "dynamical mesoscale" and grows into a mature tropical cyclone. The positions of other regimes are summarized from Riley and Lelong (2000) and Vallis (2017). 


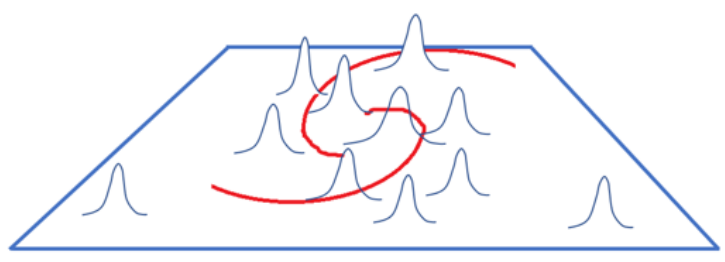

FIG. 2. A schematic diagram of the quasi-random convective seeding scheme. Convection is preferentially 1006 seeded near the high vorticity region. 

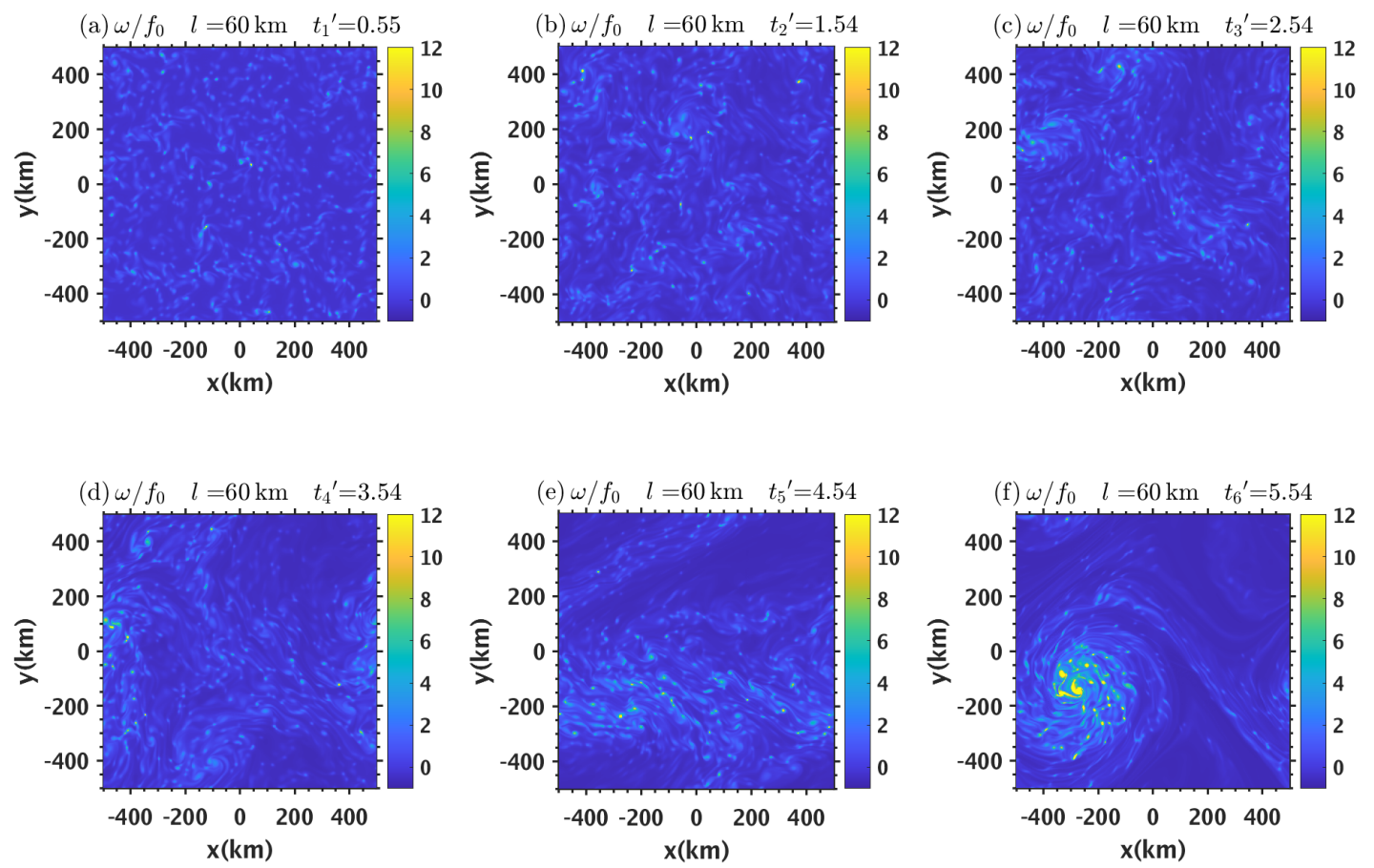

FIG. 3. The unfiltered $\omega / f_{0}$ field of the index-1-run in the ensemble of the $l=60 \mathrm{~km}$ test at (a) $t_{1}^{\prime}=-\delta_{0} t=$ 1008 0.55 , (b) $t_{2}^{\prime}=1.54$, (c) $t_{3}^{\prime}=2.54$, (d) $t_{4}^{\prime}=3.54$, (e) $t_{5}^{\prime}=4.54$, and (f) $t_{6}^{\prime}=5.54$. 

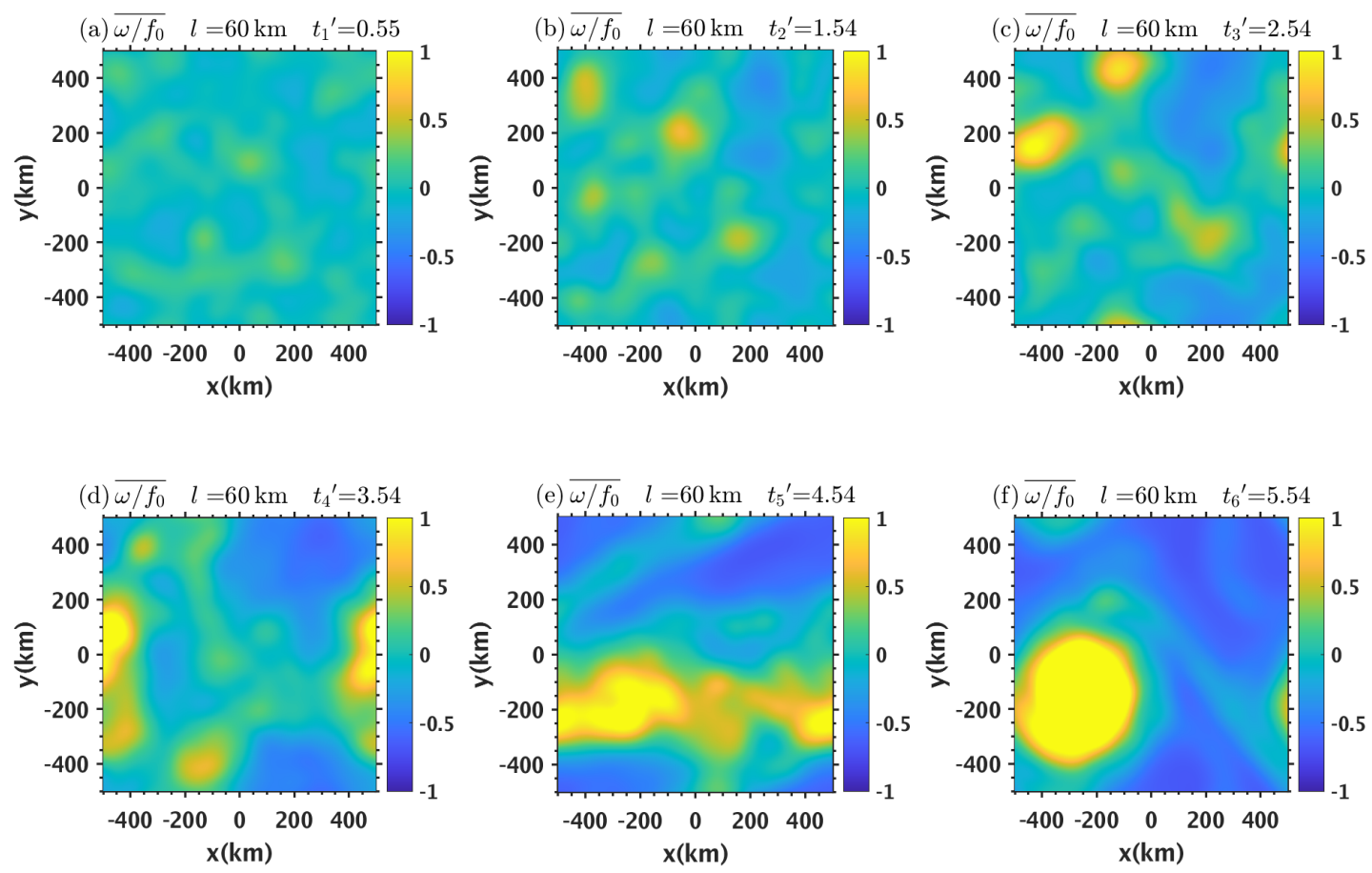

FIG. 4. The same as Fig. 3, but has been filtered with a $l=60 \mathrm{~km}$ Gaussian filter. 

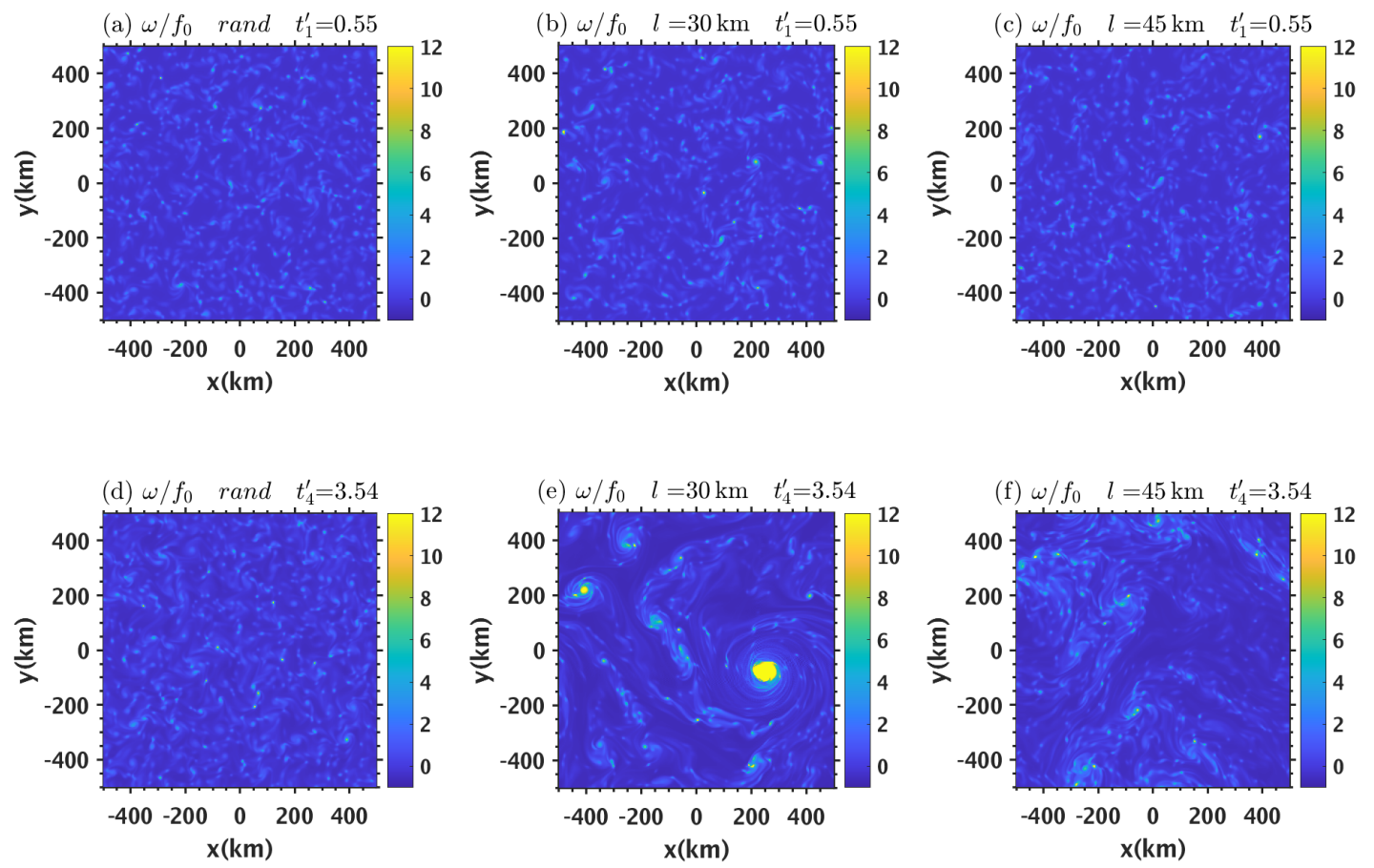

FIG. 5. (a)(b)(c) are the $t_{1}^{\prime}=0.55$ snapshots of $\omega / f_{0}$ for the purely random test, $l=30 \mathrm{~km}$ test and $l=45 \mathrm{~km}$ test, using the index-1-run in the ensemble. (d)(e)(f) are the corresponding $t_{4}^{\prime}=3.54$ vorticity snapshots. 

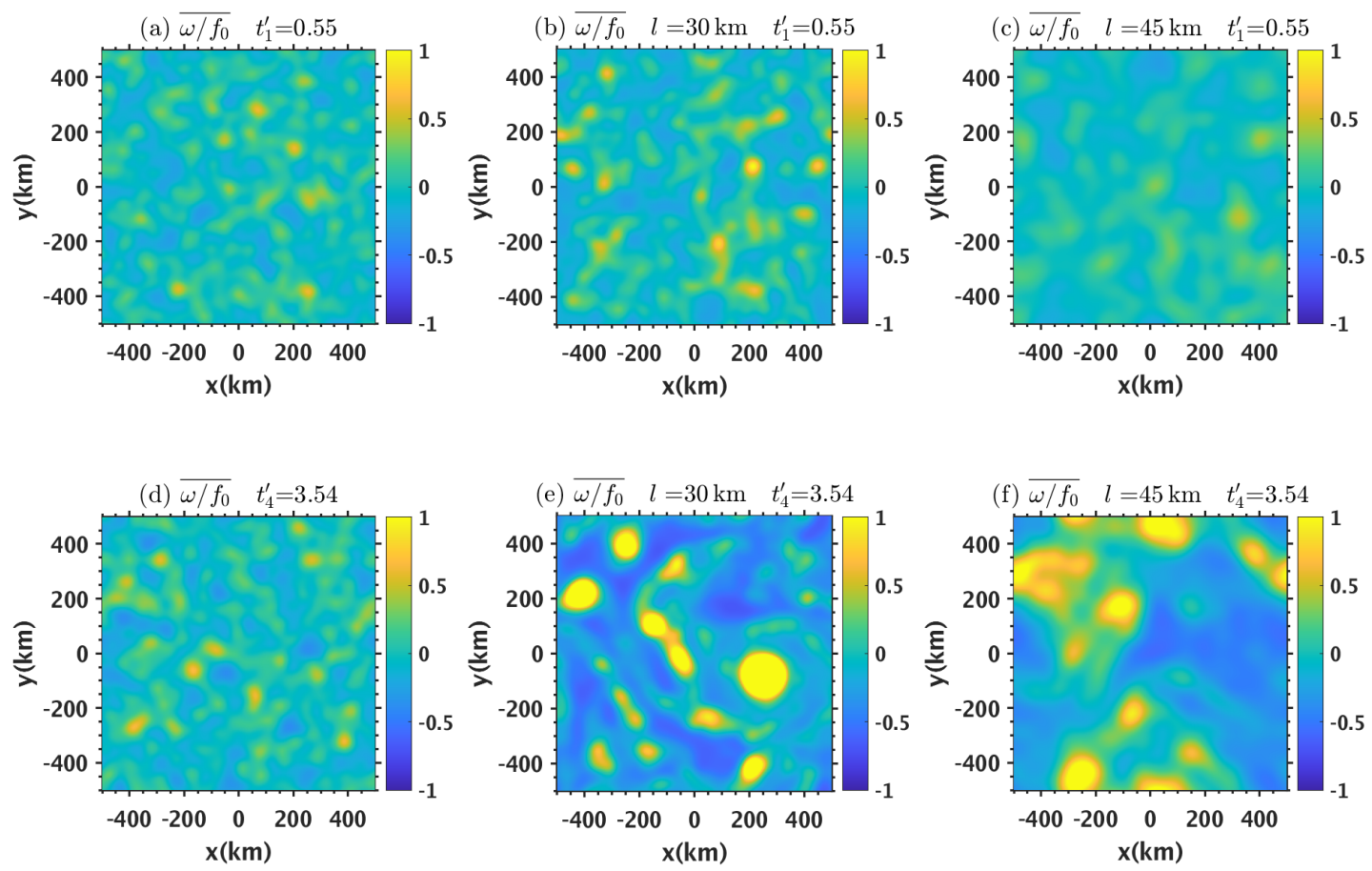

FIG. 6. The same as Fig. 5, but for the filtered field $\overline{\omega / f_{0}}$ instead. (a) and (d) are the result of the purely random test processed with a $l=30 \mathrm{~km}$ filter. (b) and (e) are the $l=30 \mathrm{~km}$ test, processed with the corresponding 1013 $l$. (c) and (f) are the $l=45 \mathrm{~km}$ test, processed with the corresponding $l$. 

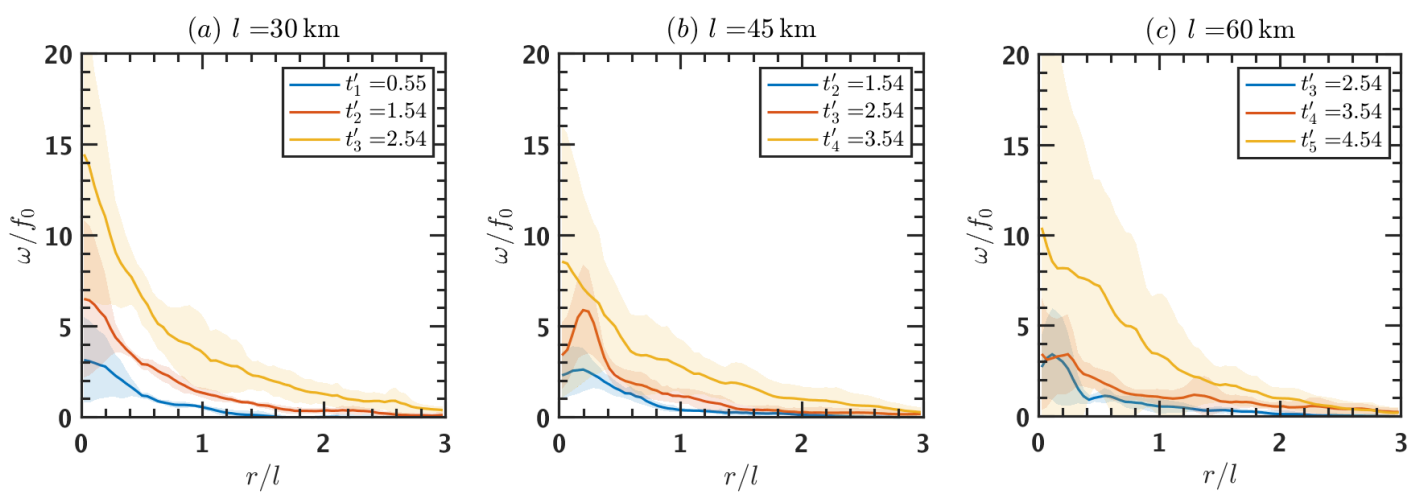

FIG. 7. The azimuthally averaged $\omega / f_{0}$ profile centered at the maximum $\bar{\omega}$ point in the domain, for (a) $l=30$ $\mathrm{km}$, (b) $l=45 \mathrm{~km}$ and (c) $l=60 \mathrm{~km}$ test respectively. The solid lines denote the ensemble-average, and the shadow denotes the \pm 1 standard deviation range. For (a), the blue, red and yellow lines denote $t^{\prime}=t_{1}^{\prime}, t_{2}^{\prime}$, and $t_{3}^{\prime}$ respectively. For (b), the blue, red and yellow lines denote $t^{\prime}=t_{2}^{\prime}, t_{3}^{\prime}$, and $t_{4}^{\prime}$ respectively. For (c), the blue, red and yellow lines denote $t^{\prime}=t_{3}^{\prime}, t_{4}^{\prime}$, and $t_{5}^{\prime}$ respectively. The sampling slot is shifted, because the system growth rate is slower for a larger $l$. 

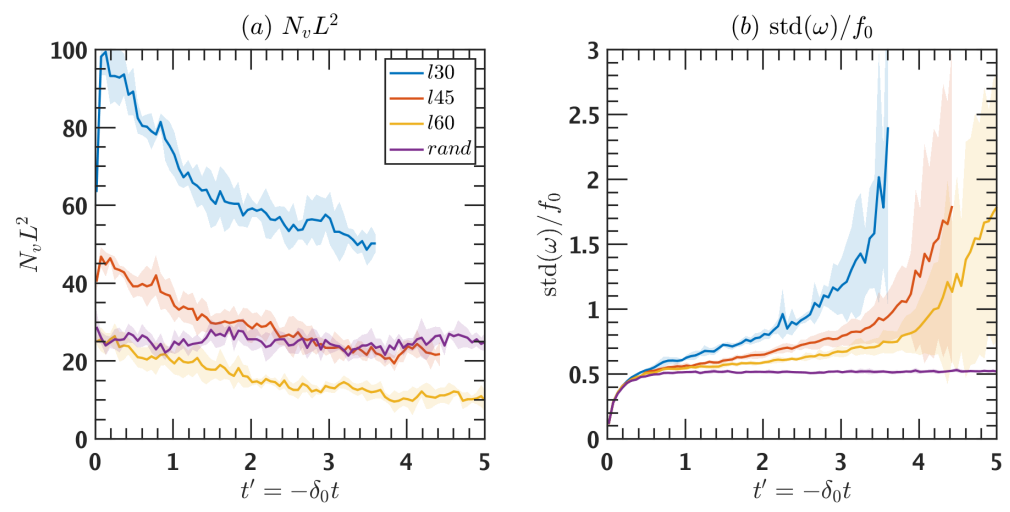

FIG. 8. (a) The total number of the maximum points in the $\bar{\omega}$ field $N=N_{v} L^{2}$. The $N_{v}$ is the spatial number density of the maximum points, which is interpreted as the number density of vortex cluster. The blue line denotes the $l=30 \mathrm{~km}$ filter test, the red line denotes the $l=45 \mathrm{~km}$ test, the yellow line denotes the $l=60 \mathrm{~km}$ test, and the purple line denotes the purely random test. The shadow denotes the \pm 1 standard deviation range of a five-member ensemble. (b) is the same as (a), but for the standard deviation of relative vorticity $\operatorname{std}(\omega)$ normalized with $f_{0}$. The time series of a test is truncated once a member in the ensemble blows up. 

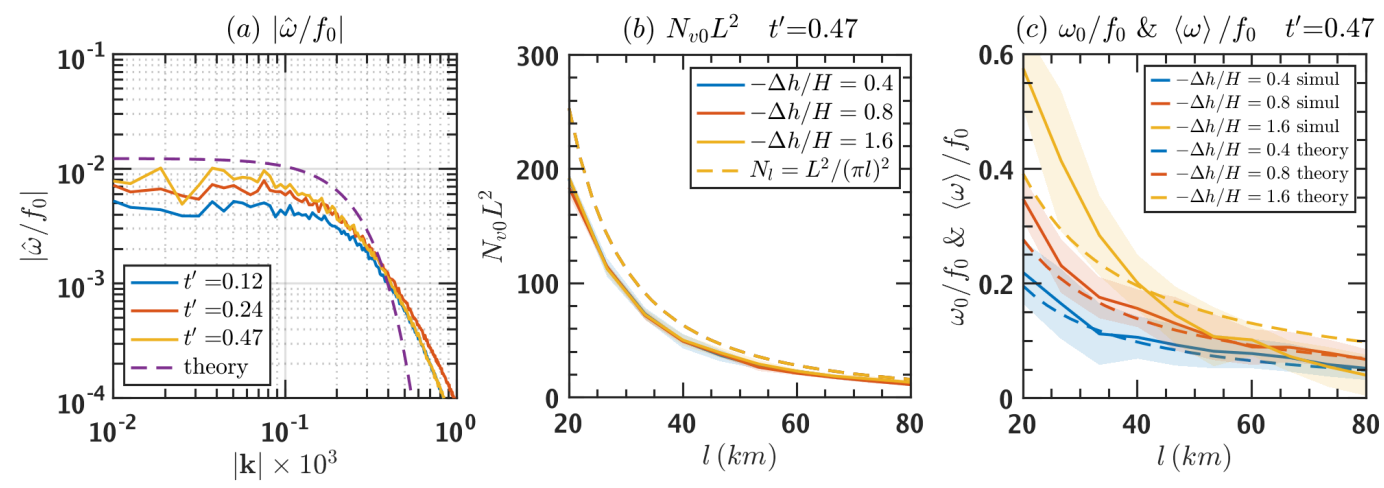

FIG. 9. (a) The one-dimensional power spectrum $|\hat{\omega}|$ of the index-1-run of the purely random test. It is obtained by "azimuthally" averaging $|\hat{\omega}|$ over each wavenumber circle (constant $|\mathbf{k}|=\sqrt{k_{x}^{2}+k_{y}^{2}}$ ). The solid blue line denotes $t^{\prime}=0.12\left(t^{\prime} \approx-\delta_{0} T_{0} / 4\right)$, the solid red line denotes $t^{\prime}=0.24\left(t^{\prime} \approx-\delta_{0} T_{0} / 2\right)$, and the solid red line denotes $t^{\prime}=0.47\left(t^{\prime} \approx-\delta_{0} T_{0}\right)$. The dashed purple line denotes the equilibrium spectrum predicted by (22). The diagnosed spectrum at the high wavenumber end is gentler than the theoretical estimate, probably due the sharpening effect of the convergent transport. (b) The dependence of domain total vortex number $N_{v 0} L^{2}$ on filter length $l$, at $t^{\prime}=0.47\left(t^{\prime} \approx-\delta_{0} T_{0}\right)$. All the simulations are run with $l=60 \mathrm{~km}$, and the value at different $l$ is due to the change of $l$ in filtering the same set of $\omega$ data. The solid blue, red and yellow lines denote the data using $-\Delta h / H=0.4,0.8$ and 1.6 respectively. Note that $\tau_{u}$ is changed proportionally to keep the updraft-induced convergence $-\Delta h /\left(\tau_{u} H\right)$ unchanged. The yellow dashed line denotes the theoretical prediction $N_{l}=L^{2} / \pi l^{2}$. (c) is the same as (b), but for benchmarking the theoretical $\omega_{0} / f_{0}$ at $-\Delta h / H=0.4,0.8$ and 1.6 (dashed blue, red and yellow lines). The simulation counterpart (solid lines) uses $\langle\omega\rangle / f_{0}$, where $\langle\omega\rangle$ is defined as the area average within $2 l$ radius of the maximum $\bar{\omega}$ point (the strongest vortex cluster). The motivation of this choice is explained in section 3c. 

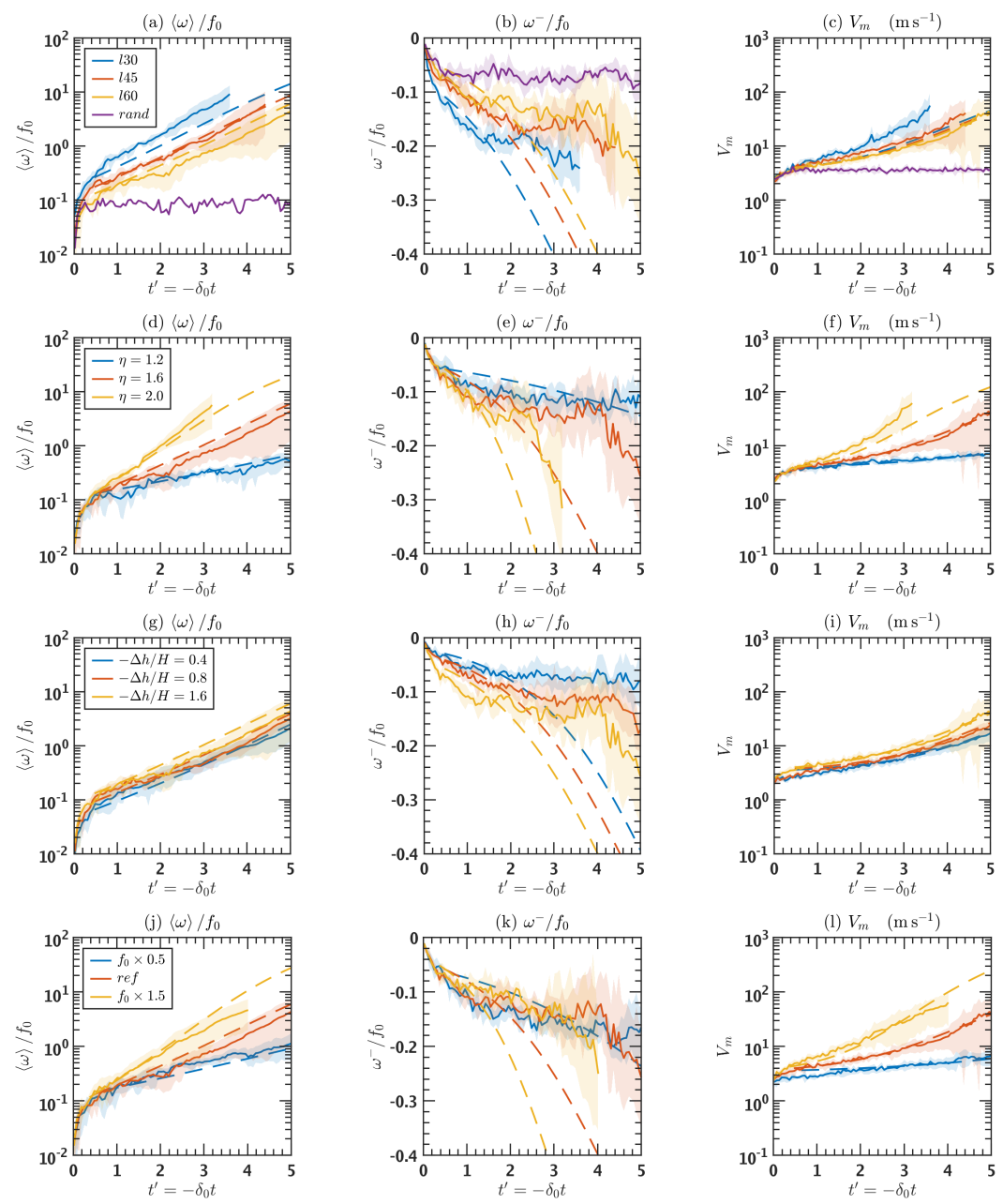

FIG. 10. The comparison between the barotropic simulation (denoted as the solid lines) and the theory (the numerical solution of the ODE system, denoted as the dashed lines) on the time series of $\langle\omega\rangle / f_{0}$ (the left column), $\omega^{-} / f_{0}$ (the middle column) and the maximum wind $V_{m}$ (the right column). The first row (a)(b)(c) is for different $l$, with the blue, red, and yellow lines denoting the five-run ensemble average of $l=30 \mathrm{~km}, l=45$ $\mathrm{km}, l=60 \mathrm{~km}$ and purely random test (equivalent to $l \rightarrow \infty$ ) respectively. The shadow denotes the corresponding \pm 1 standard deviation range of the barotropic simulation. The second row (d)(e)(f) is for $\eta=1.2$ (the blue line), $\eta=1.6$ (the red line, also the reference test), and $\eta=2$ test (the yellow line). The third row (g)(h)(i) is for $-\Delta h / H=0.4$ (the blue line), 0.8 (the red line), and 1.6 test (the yellow line, also the reference test). The forth row $(\mathrm{j})(\mathrm{k})(\mathrm{l})$ is for the half Coriolis parameter $f_{0}=0.5 \times 10^{-4}$ test (the blue line), the reference test (the red line), and the 1.5 times Coriolis parameter $f_{0}=1.5 \times 10^{-4} \mathrm{~s}^{-1}$ test (the yellow line). The time series of a test is truncated once a member in the ensemble blows up. 

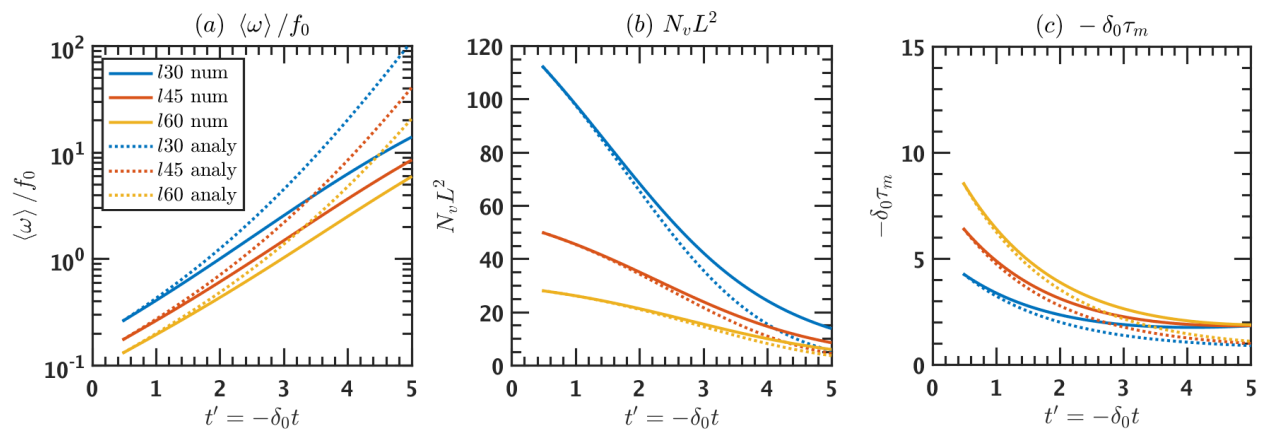

FIG. 11. (a) The evolution of $\langle\omega\rangle / f_{0}$ predicted by the theory for $l=30,45$ and $60 \mathrm{~km}$ tests, which are denoted as the blue, red and yellow lines respectively. The solid lines denote the numerical solution of the ODE system, and the dashed lines denote the approximate analytical solution. (b) is the same as (a), but for the total vortex number in the domain $N_{v} L^{2}$. (c) is the same as (a), but for the nondimensional merger timescale $-\delta_{0} \tau_{m}$. 

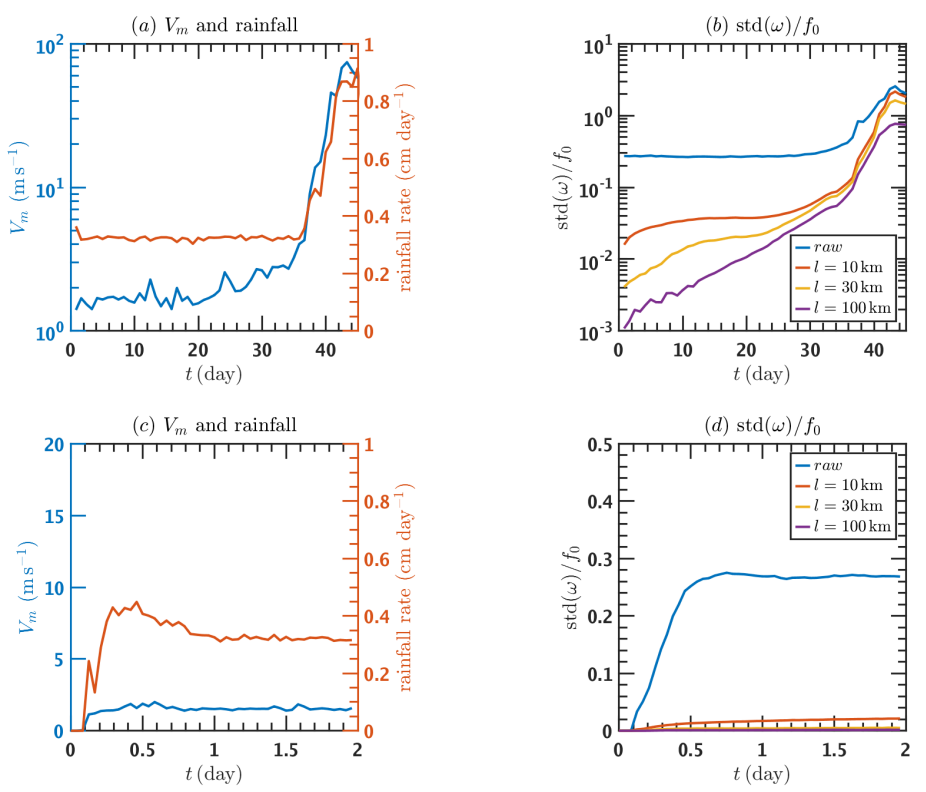

FIG. 12. (a) The blue line denotes the low-mid level maximum total wind calculated using the $1.18-6.25 \mathrm{~km}$ vertically averaged velocity vector, and the red line denotes the domain-average rainfall rate (unit: $\mathrm{cm} \mathrm{day}^{-1}$ ). (b) The standard deviation of low-mid level vertical vorticity $\omega$ (averaged between $1.18-6.25 \mathrm{~km}$ height). The blue line denotes the domain-average time series. The red, yellow and purple lines denote the standard deviation of the vorticity filtered with $l=10 \mathrm{~km}, 30 \mathrm{~km}$ and $100 \mathrm{~km}$. (c) and (d) are the zoom-in plots of (a) and (b) for the first two days. 

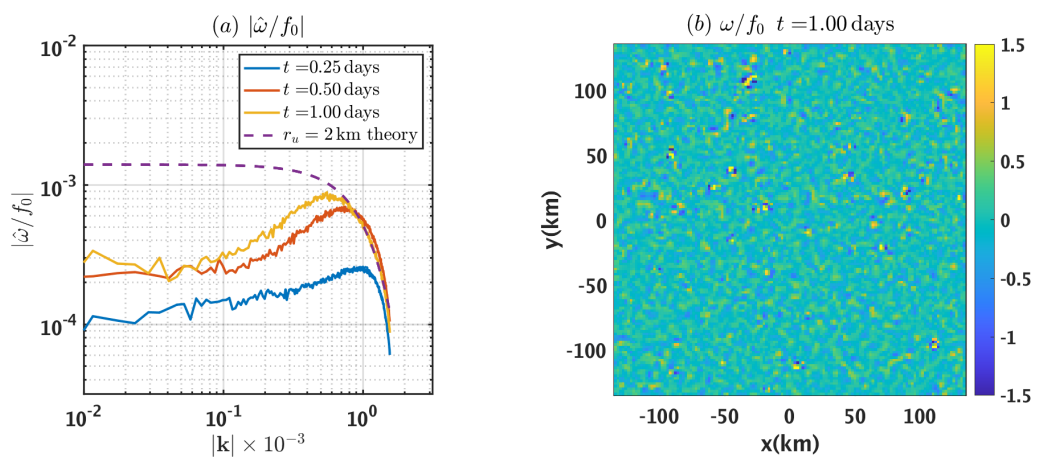

FIG. 13. (a) The power spectrum of $\omega / f_{0}$ at $t=0.25$ days (the blue line), $t=0.50$ days (the red line), and $t=1.00$ days (the yellow line). It is calculated in the same way as Fig. 9a. The dashed purple line is a theoretical estimation of the equilibrium spectrum using (22), with the parameters listed in section $6 \mathrm{~d}$ (e.g. $\left.r_{u}=2 \mathrm{~km}\right) .(\mathrm{b})$ A zoom-in plot of the central region $\omega / f_{0}$ at $t=1.00$ days. 

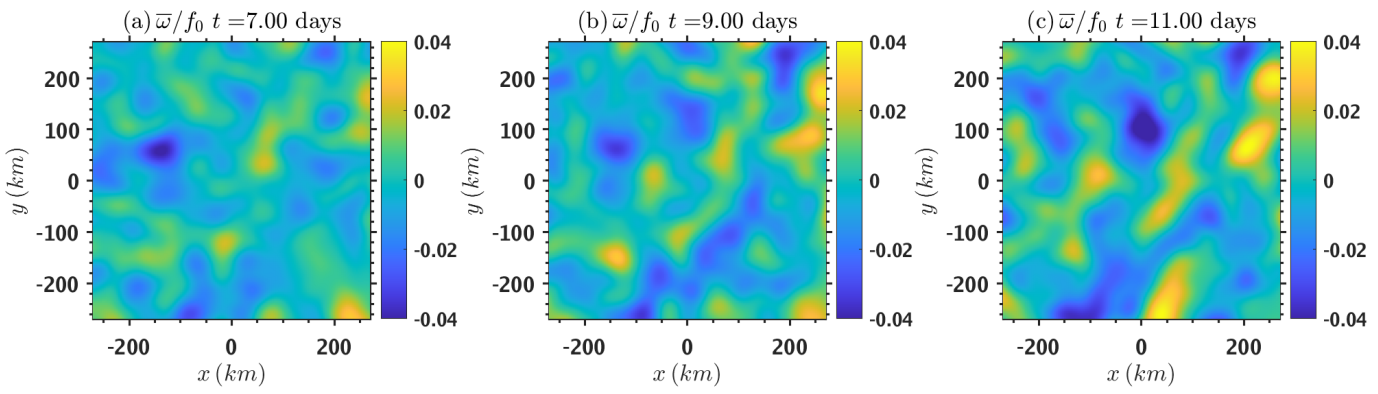

FIG. 14. A zoom-in plot of the $\bar{\omega} / f_{0}$ which uses a $30 \mathrm{~km}$ Gaussian filter, at (a) $t=7$ days, (b) $t=9$ days, and 1066 (c) $t=11$ days. They show the stationary growth sub-regime in the deterministic regime. 

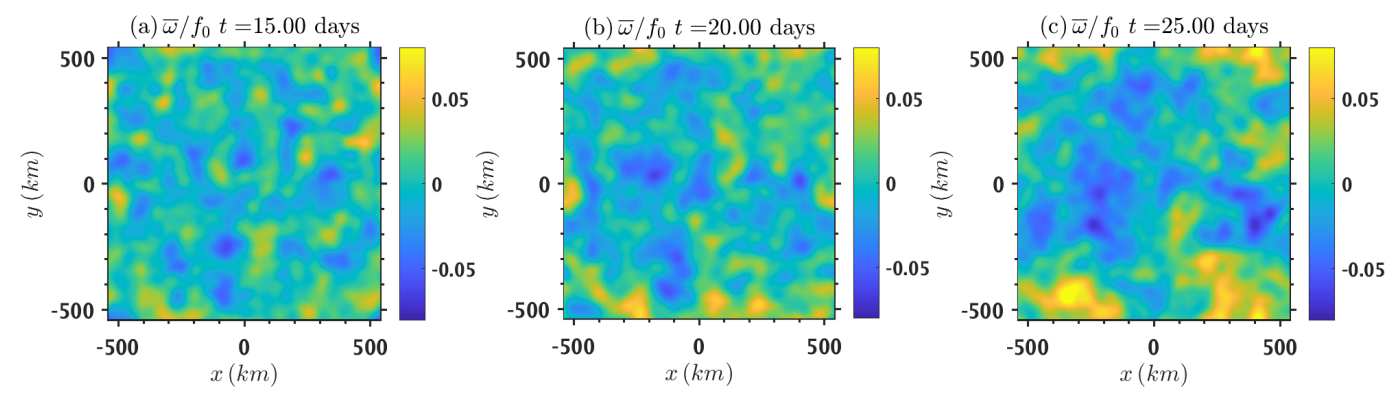

1067

FIG. 15. A plot of the $\bar{\omega} / f_{0}$ which uses a $30 \mathrm{~km}$ Gaussian filter, at (a) $t=15$ days, (b) $t=20$ days, and (c) ${ }_{1068} t=25$ days. They show the merger sub-regime in the deterministic regime. 

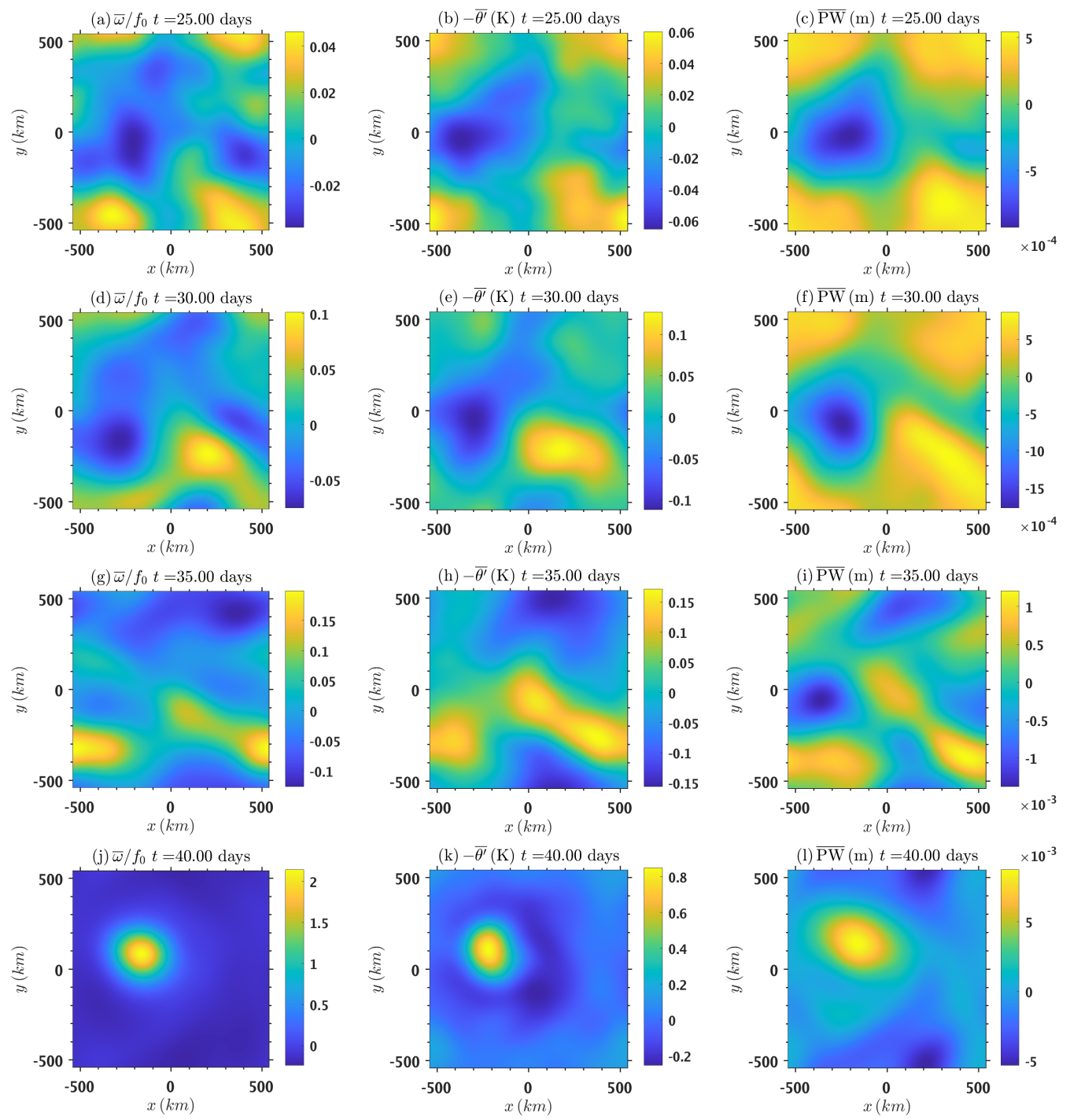

FIG. 16. The $100 \mathrm{~km}$-Gaussian filtered quantities. The first column denotes the nondimensional filtered lowmid level vertical vorticity $\left(\bar{\omega} / f_{0}\right)$. The second column denotes the opposite of the filtered disturbance potential temperature $-\overline{\theta^{\prime}}$ at $z=25 \mathrm{~m}$ (having subtracted the domain-averag value). The third column denotes the filtered column precipitable water (PW, unit: $\mathrm{m}$ ). The first, second, third and fourth row denotes the data at $t=25$ days, 30 days, 35 days and 40 days respectively. The range of the colorbar is not controlled, because we focus on the pattern. 

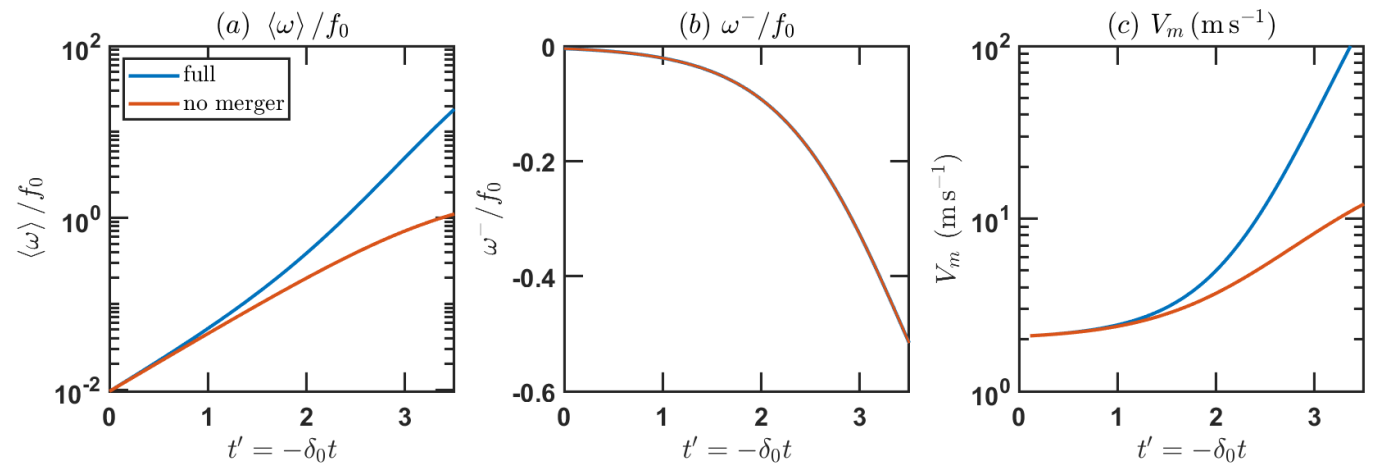

FIG. 17. The numerical solution of the ODE system of the mixed stochastic-deterministic theory. (a) The characteristic vorticity of the vortex cluster $\langle\omega\rangle / f_{0}$, (b) the environmental vorticity $\omega^{-} / f_{0}$, and (c) the maximum wind $V_{m}$. The red line denotes the numerical solution that arbitrarily turns off the merger term (by setting 1078 $\left.\alpha_{m} \rightarrow \infty\right)$, and the blue line denotes the normal solution with the merger term. Note that the $\omega^{-} / f_{0}$ of the two simulations is identical. 


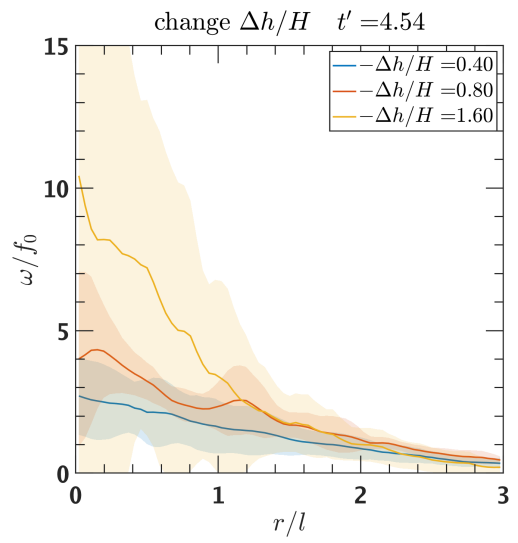

${ }_{1080}$ Fig. A1. The azimuthal average $\omega / f_{0}$ profile centered at the maximum $\bar{\omega}$ point at $t_{5}^{\prime}=4.54$. The blue line 1081 denotes the five-member ensemble average of the $-\Delta h / H=0.4$ test, the red line denotes the $-\Delta h / H=0.8$ test, 1082 and the yellow line denotes the $-\Delta h / H=1.6$ test (reference test). The shadow of corresponding color denotes 1083 the \pm 1 standard deviation. 